\title{
On the Utility of Antiprotons as Drivers for Inertial Confinement Fusion
}

L. John Perkins, Charles D. Orth, Max Tabak - Lawrence Livermore National Laboratory

\section{June 15, 2003}

U.S. Department of Energy

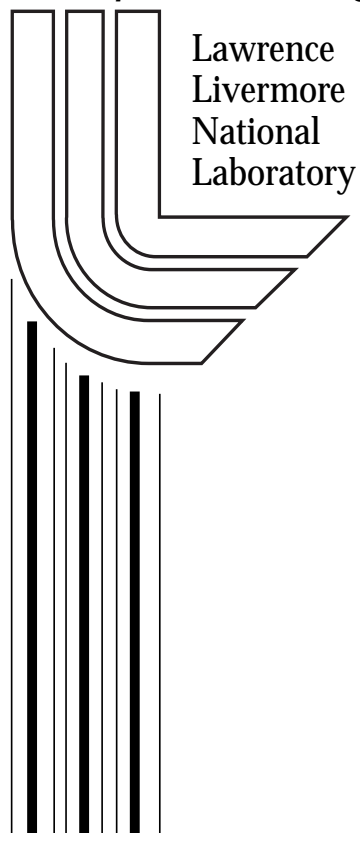




\section{DISCLAIMER}

This document was prepared as an account of work sponsored by an agency of the United States Government. Neither the United States Government nor the University of California nor any of their employees, makes any warranty, express or implied, or assumes any legal liability or responsibility for the accuracy, completeness, or usefulness of any information, apparatus, product, or process disclosed, or represents that its use would not infringe privately owned rights. Reference herein to any specific commercial product, process, or service by trade name, trademark, manufacturer, or otherwise, does not necessarily constitute or imply its endorsement, recommendation, or favoring by the United States Government or the University of California. The views and opinions of authors expressed herein do not necessarily state or reflect those of the United States Government or the University of California, and shall not be used for advertising or product endorsement purposes.

This work was performed under the auspices of the U. S. Department of Energy by the University of California, Lawrence Livermore National Laboratory under Contract No. W-7405-Eng-48.

This report has been reproduced directly from the best available copy.

Available to DOE and DOE contractors from the

Office of Scientific and Technical Information

P.O. Box 62, Oak Ridge, TN 37831

Prices available from (423) 576-8401

http://apollo.osti.gov/bridge/

Available to the public from the National Technical Information Service

U.S. Department of Commerce 5285 Port Royal Rd., Springfield, VA 22161

http://www.ntis.gov/

OR

Lawrence Livermore National Laboratory

Technical Information Department's Digital Library

http://www.llnl.gov/tid/Library.html 


\title{
On the Utility of Antiprotons as Drivers for Inertial Confinement Fusion
}

\author{
L. John Perkins, Charles D. Orth, Max Tabak \\ Lawrence Livermore National Laboratory
}

June 15, 2003

\begin{abstract}
By contrast to the large mass, complexity and recirculating power of conventional drivers for inertial confinement fusion (ICF), antiproton annihilation offers a specific energy of $90 \mathrm{MJ} / \mu \mathrm{g}$ and thus a unique form of energy packaging and delivery. In principle, antiproton drivers could provide a profound reduction in system mass for advanced space propulsion by ICF. We examine the physics underlying the use of antiprotons $(\bar{p})$ to drive various classes of high-yield ICF targets by the methods of volumetric ignition, hotspot ignition and fast ignition. The useable fraction of annihilation deposition energy is determined for both $\overline{\mathrm{p}}$-driven ablative compression and $\overline{\mathrm{p}}$-driven fast ignition, in association with $0-\mathrm{D}$ and 1-D target burn models. Thereby, we deduce scaling laws for the number of injected antiprotons required per capsule, together with timing and focal spot requirements. The kinetic energy of the injected antiproton beam required to penetrate to the desired annihilation point is always small relative to the deposited annihilation energy. We show that heavy metal seeding of the fuel and/or ablator is required to optimize local deposition of annihilation energy and determine that a minimum of $\sim 3 \times 10^{15}$ injected antiprotons will be required to achieve high yield (several hundred megajoules) in any target configuration. Target gains - i.e., fusion yields divided by the available $p-\bar{p}$ annihilation energy from the injected antiprotons $(1.88 \mathrm{GeV} / \overline{\mathrm{p}})-$ range from $\sim 3$ for volumetric ignition targets to $\sim 600$ for fast ignition targets. Antiproton-driven ICF is a speculative concept, and the handling of antiprotons and their required injection precision - temporally and spatially - will present significant technical challenges. The storage and manipulation of low-energy antiprotons, particularly in the form of antihydrogen, is a science in its infancy and a large scale-up of antiproton production over present supply methods would be required to embark on a serious R\&D program for this application.
\end{abstract}




\section{CONTENTS}

1. INTRODUCTION

2. ANTIPROTON ANNIHILATION

3. TARGET CONFIGURATIONS

3.1 Inertial Confinement Physics

3.2 Antiproton Volumetric Ignition

3.3 Antiproton Hotspot Ignition

3.4 Antiproton Fast Ignition

3.5 Muon Catalysis

4. ANTIPROTON-DRIVEN FISSION-FUSION VOLUMETRIC IGNITION

4.1 Analysis Method

4.2 Burn Calculations

4.3 Antiproton Energy Deposition

4.4 Performance Parametrics

4.5 Volumetric Ignition Design Point

5. ANTIPROTON-DRIVEN HOTSPOT IGNITION

5.1 Hotspot Ignition Physics

5.2 Analysis and Results

5.3 Hotspot Ignition Design Point

5.4 Target Stability

6. ANTIPROTON-DRIVEN FAST IGNITION

6.1 Fast Ignition Rationale

6.2 Fast Ignition Hotspot Creation

6.3 Slow Compression Drive

6.4 Fast Ignition Design Point

7. TIMING AND AIMING REQUIREMENTS FOR ANTIPROTON INJECTION

8. ANTIPROTON PRODUCTION, STORAGE AND INJECTION

9. SUMMARY AND CONCLUSIONS

10. REFERENCES

\section{Acknowledgements}

The authors take pleasure in acknowledging Jay Polk and Stephanie Leifer of JPL/NASA for encouraging our explorations in this topic; Gerald Smith, formally of Pennsylvania State University, for directing us to his earlier concepts in the field; David Morgan and Mark Herrmann of Lawrence Livermore National Laboratory for informative discussions on antiproton physics and indirect-drive ICF capsules, respectively.

This work was performed under the auspices of the U. S. Department of Energy by the University of California, Lawrence Livermore National Laboratory, under Contract No. W-7405-Eng-48. The research was funded under the Work for Others Proposal \#L8750 by the National Aeronautics and Space Administration (NASA) through the Jet Propulsion Laboratory (JPL). 


\section{INTODUCTION}

In inertial confinement fusion (ICF), a driver - e.g., a laser, heavy-ion accelerator or pulse-power z-pinch - delivers an intense pulse of energy to a target containing a few milligrams of fusion fuel, typically deuterium-tritium. During the short time for the fuel to compress to very high densities and temperatures, the inertia of the fuel mass provides the confinement necessary to achieve thermonuclear burn [1,2]. A number of studies have examined the application of such conventionally driven ICF as both an energy source for terrestrial electric power generation [1,3-6] and for advanced space propulsion [7-9]. Depending on the driver type and fuel ignition method, driver energies in the range of several hundred kilojoules to several megajoules will be required to achieve high fusion energy gain from an ICF target $[1,2,10,11]$. Conventional drivers producing this total energy are massive, complex and, even in extrapolated reactor projections with optimistic assumptions of cost and performance, will likely cost a good fraction of a billion dollars $[1,3-6,12]$. For space propulsion by ICF, the total mass of the driver system is of paramount concern and includes the driver itself, power supplies, the recirculating energy supply system and associated waste heat radiators (see, for example, Ref. 9).

By contrast to the large system masses of conventional ICF drivers, antiprotons offer a unique form of energy packaging and delivery. With the highest specific energy for any physical entity, an incident antiproton beam presents an injected energy of $90 \mathrm{MJ} / \mu \mathrm{g}$ when annihilating with an equal mass of normal matter - some 270 times greater than the specific energy of deuterium-tritium fusion fuel. Put in perspective, the energy content of one microgram of delivered antiprotons (plus an equal mass of normal matter) is equivalent to one hundred full power shots from the National Ignition Facility (NIF), a laser-driven facility presently under construction and designed to achieve ICF ignition and thermonuclear energy gain* [13]. Thus, in principle, antiproton drivers could provide a profound reduction in both "wet" and "dry" masses for advanced space propulsion by ICF. For example, as described below, a few moles of onboard, stored antiprotons and an appropriate low energy injection system could comprise the complete driver system for an ICF-powered spacecraft delivering a 100tonne payload to Mars. Of course, from the viewpoint of $\overline{\mathrm{p}}$ production today, this is an enormous number of antiprotons.

Whether such drivers have any utility to terrestrial electric power production by inertial fusion will ultimately depend on the projected efficiency of antiproton production, collection and storage. By contrast, for space-based applications, the production efficiency of antiprotons in external facilities is of lower consequence

* NIF [13] offers a real example of a conventional ICF driver designed to deliver 1.8MJ of laser light to a DT capsule. The facility is the scale of a sports stadium with a total mass in excess of 100,000tonnes and total project cost of $\sim \$ 2.3 \mathrm{~B}$. Note that in assessing total mass, NIF would also be implicitly burdened with the external electricity supply system required to charge the capacitor banks between every shot. In an ICF reactor with net energy gain, a portion of the output fusion energy would be recycled via the onsite/onboard electricity generating plant to resupply the driver but at the expense of extra plant mass and, in space, additional heat radiator mass. In the case of antiproton drive, nearly all this mass and complexity could be eliminated. 
compared to their on-board energy storage potential. Overall energy breakeven is not necessarily required.

In this paper, we examine the application of antiprotons to drive ICF targets of various classes including volumetric ignition, hotspot ignition and fast ignition. Relative to inertial confinement driven by conventional methods and, in particular, from the space propulsion viewpoint, the following potential advantages may pertain:

- A vast reduction in driver mass, recirculating power hardware and waste heat radiators

- Minimal or zero need for recirculating power (auxiliary needs in space could be fulfilled by a small, separate fission power source)

- Very low injection energies (driver energy originates from deposited annihilation energy and not the kinetic energy of the beam)

- Ability to channel through target blowoff debris by adjustment of the delivery kinetic energy.

We caution that although antiprotons potentially offer a novel, high-energy-density packaging scheme, their application to antiproton-driven fusion is speculative. In particular, both the handling of antiprotons and their required injection precision - both in space and time - will present substantial technical challenges. Moreover, the production, storage and manipulation of antiprotons at low energy, particularly in the form of anti(neutral)hydrogen, is a science in its infancy [14-20]. Finally, a large scale-up of antiproton production over present supply methods would be required.

The concept of employing antiprotons to drive micro-fission assemblies was proposed in 1973 by Askaryan et al [21] and Winterberg [22]. Smith [23] reported initial investigations of antiproton-driven fission in uranium on the Low Energy Antiproton Ring at CERN in 1987; these experiments were discussed by Angelopoulos et al. [24]. Machner [25] also measured anti-proton-induced fission in uranium. Lewis et al [26] extended the analysis of antiproton-boosted micro-fission and considered candidate target designs and potential applications. Similarly, Gazze et al [27] and Kumiko et al [28] studied pion production/transport, and neutron production/transport, respectively, in such compressed targets. Von Egidy [29] et al. and Schmid et al. [30] employed a fission fragment spectrometer to determine absolute antiproton-induced fission yields in ${ }^{238} \mathrm{U}$, ${ }^{232} \mathrm{Th},{ }^{209} \mathrm{Bi},{ }^{197} \mathrm{Au}$ and ${ }^{165} \mathrm{Ho}$. Gsponer and Hurni [31, 32] considered the use of antihydrogen for triggering ICF pellets and larger thermonuclear explosions.

In 1990, Lewis et al [33] proposed using antiproton-induced fission to boost the burning of fusion fuel while Smith [34] proposed the analogous Ion-Compressed Antimatter Nuclear (ICAN) concept for antiproton-catalyzed fission-fusion propulsion. Studies to analyze such concepts were proposed by Beller and Martin [35] and potential propulsion performance was scoped by Nugent [36] and Wienke [37]. Similarly, Gaidso [38] proposed Antimatter Initiated Microfusion (AIM) for deep space missions where D${ }^{3} \mathrm{He}$ fusion fuel would be injected into an antiproton cloud confined in a 20T Penning trap. As far as the present authors are aware, none of these papers have formally assessed 
the integrated energy balance in the respective targets from antiproton deposition through fission and fusion energy release. In addition, none have proposed using antiprotons to ablatively implode ICF hotspot or fast-ignition targets in a manner analogous to that of conventional radiation-driven or laser-driven capsules.

Many studies have proposed space propulsion with pure antimatter (see, for example, Refs. 39-48) where the energy of the $\mathrm{p}-\overline{\mathrm{p}}$ annihilation products is either used directly in the form of magnetically vectored thrust at high exhaust velocities [42-46] or, more simply, employed to heat a hydrogen working fluid $[41,47,48]$. By contrast, in the concepts of this paper, the antiproton annihilation energy is used only to drive an ICF target so that the majority of the propulsion energy would originates from the fusion process. Pure antimatter is capable of producing the highest intrinsic exhaust velocities of any known propulsion fuel at high thrust and its potential is a factor of ten or more superior in this regard compared even to fusion. However, relative to antiproton-driven ICF, pure antimatter propulsion has two disadvantages. First, much more stored antimatter is required per unit of energy expended; the mass ratios scale approximately inversely with the fusion gain of the ICF target. Second, the Brillouin limit - that is, the space charge equilibrium condition - sets a limit on the density of antiprotons stored as non-neutral, single ions in a confining magnetic trap. This will be considered further below, but the consequence is that the energy stored in the magnetic field will always be greater than the total rest mass energy of the antimatter so confined. Thus, for pure $\mathrm{p}-\overline{\mathrm{p}}$ propulsion with no fusion present, energy rationalization would dictate antiproton storage in some - and, as yet, unknown - form of neutral antihydrogen.

The purpose of this paper is to assess the conditions under which antiprotons could be employed to drive ICF targets to ignition and high gain. In particular, we will determine the useable deposition fraction of annihilation energy together with integrated energy balance in various classes of ICF targets and, thereby, deduce the typical number of injected antiprotons required. Although the target physics will be emphasized rather than practical systems aspects, the application viewpoint will be relative to space propulsion. Note that by present estimates, the minimum energy that must be absorbed in any ICF fuel capsule to promote ignition and burn is in the vicinity of $100 \mathrm{~kJ}$ [2]. Thus even with an (improbable) $100 \%$ deposition efficiency of the $1.88 \mathrm{GeV}$ energy available from each antiproton annihilation, a minimum number of $\sim 3 \times 10^{14}$ antiprotons would be required to drive a candidate target.

\section{ANTIPROTON ANNIHILATION}

On entering normal matter, the initial kinetic energy of a directed beam of antiprotons is transferred to the electrons of the medium through electron drag and the antiproton slows down similar to a conventional (positively-charged) heavy particle. At an energy of $\sim 10-25 \mathrm{keV}$, the (negative) antiproton will displace an outer orbital electron and be captured by an atom in the medium, forming an "antiprotonic atom". The antiproton cascades down towards the ground state by the emission of x-rays and reaches an inner stable Bohr orbit of radius $m_{e} / m_{p}$ smaller than the conventional electron orbit. It annihilates from this bound state with either a proton or neutron from within the nucleus to which it is attached [49]. The lifetime for this bound state is very short, i.e., $<10^{-12} \mathrm{~s}$ and the initial annihilation energy released is equal to $\sim 1.88 \mathrm{GeV}$, twice the proton rest mass. 
A key question here is what fraction of this energy is usefully captured in the fuel and/or ablator of an ICF target?

Antiprotons can also undergo direct annihilation with a nucleus in flight at kinetic energies below a few tens of $\mathrm{MeV}$, although cross sections for this are small relative to electron drag and subsequent antiprotonic atom formation [50]. Therefore, directed antiprotons annihilate at the end of their range and the stopping point could be selected in an ICF target via control of their initial kinetic energy to within the straggling range distribution.

The annihilation of antiprotons is a strong interaction process that takes place at the level of the quark structure of the nucleus. Antiproton annihilation with an isolated proton at rest is shown in Fig. 1, where we indicate typical average number of daughter particles produced. The division of the $1.88 \mathrm{GeV}$ annihilation energy is, initially, $\sim 64 \%$ to the kinetic energy of the pions and $\sim 36 \%$ to their rest energy. In $\sim 5 \%$ of annihilation events, a kaon pair is also emitted. These meson products are all unstable, thus several decay chains subsequently occur as shown in the figure. The neutral pion $\left(\pi^{\mathrm{o}}\right)$ with its very short lifetime, travels less than a micron before decaying into two energetic gamma rays. Most of this gamma energy will escape the capsule. The charged pions $\left(\pi^{ \pm}\right)$decay into muons $\left(\mu^{ \pm}\right)$and neutrinos $\left(v_{\mathrm{e}, \mathrm{m}}\right)$. The muons then further decay into electrons $\left(\mathrm{e}^{-}\right)$ and positrons $\left(\mathrm{e}^{+}\right)$, and the latter subsequently annihilate with electrons in the surrounding medium.

Due to the different quark structures of protons and neutrons ${ }^{*}$, the reaction products from antiproton annihilation with an isolated neutron are slightly different from those shown above with an isolated proton. In the case of neutrons, the mean number of negative pions is one greater than the mean number of positive pions and the ratio of charged to neutral pions is somewhat greater [49].

The above particle and energy distributions apply to annihilation of an antiproton with a single, isolated proton (i.e., hydrogen nucleus) or neutron. Annihilation with a normal matter nucleon within a heavy nucleus of $A \gg>1$ produces rather different products and energetics. A fraction of available annihilation energy is transferred to the heavy nucleus via pion interactions as shown in Fig. 2. The nucleus can break up in a "super fission" process resulting in highly ionizing, short-range nuclear fragments. In such case, the total energy released can be more than the $1.88 \mathrm{GeV}$ from annihilation due to the fission contribution - i.e., the repulsive Coulomb energy. Experimental results from Smith [23], Angelopoulos et al. [24] and Machner et al. [25] claim that annihilation with uranium induces conventional binary fission in $\sim 100 \%$ of the events, while Schmid et al [30] obtains only $77 \%$ binary fissions with the balance to multiple lighter ${ }^{238} \mathrm{U}$ fragments. Note also that because the critical energy to promote fission in heavy, non-

\footnotetext{
* The proton is composed of two "up" quarks, each of charge $+2 / 3$, plus one "down" quark of charge $-1 / 3$. The neutron is composed of two down quarks plus one up quark, while the antiproton has two anti-up quarks (charge -2/3) plus one anti-down quark (charge $+1 / 3$ ). The mesons resulting from the annihilation reactions are composed of quark-antiquark pairs; e.g. the positive pion is composed of an up quark (charge $+2 / 3$ ) and an anti-down quark (charge $+1 / 3$ ).
} 


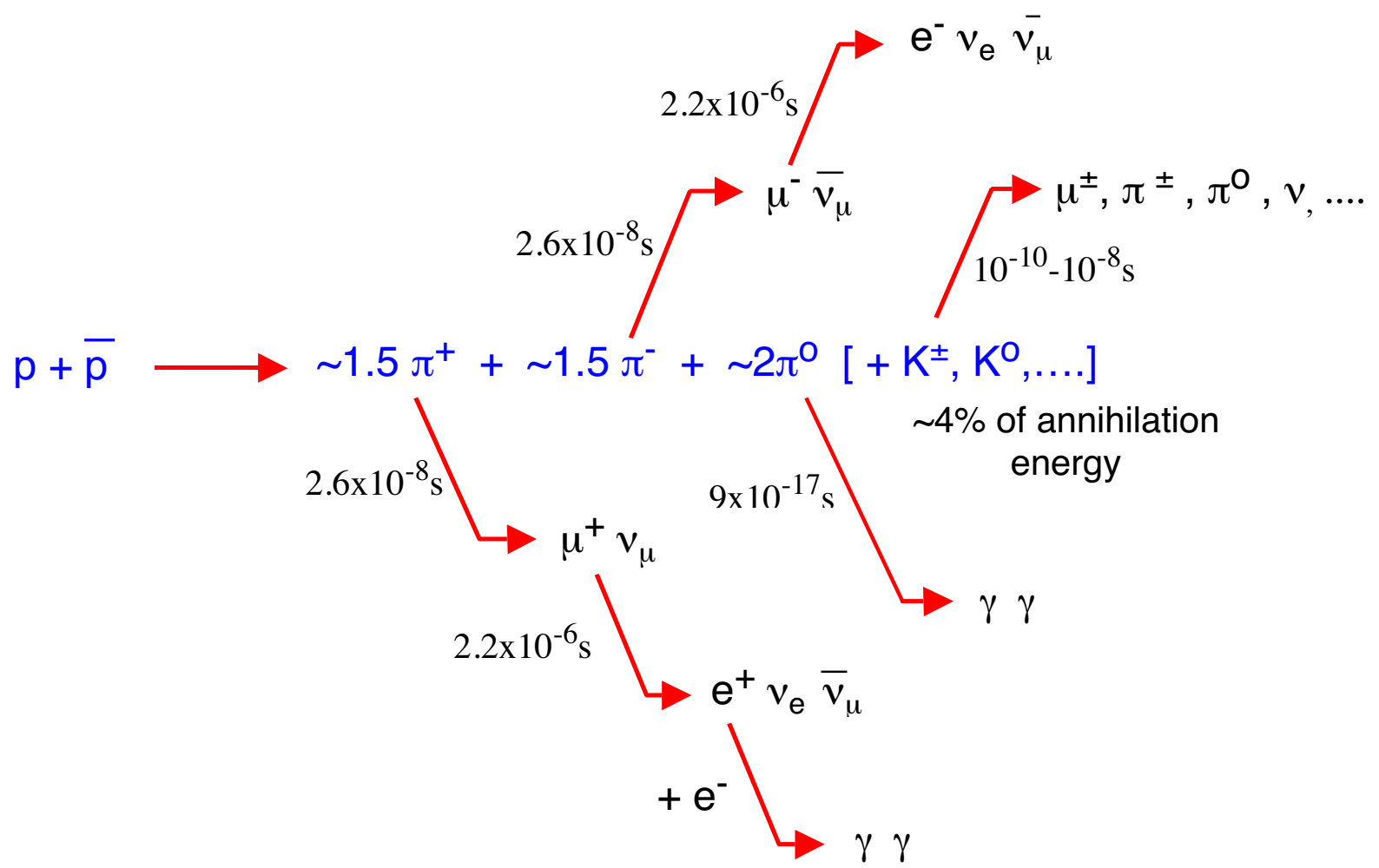

Fig. 1. Typical products resulting from annihilation of an antiproton with an isolated proton. Annihilation with an isolated neutron is similar except for a change in the ratio of charged and neutral pions. Intrinsic particle lifetimes are shown; mean lifetimes in the lab frame are $\sim 7 \times 10^{-8}$ s for $\pi^{ \pm}$decay and $\sim 6 \times 10^{-6}$ s for $\mu^{ \pm}$decay

fissile nuclei ranges from a few $\mathrm{MeV}$ in ${ }^{238} \mathrm{U}$ and ${ }^{232} \mathrm{Th}$ to tens of $\mathrm{MeV}$ for elements tungsten through bismuth, multiple fragment breakup can occur when antiprotons annihilate in such nuclei'.

In the candidate target designs described below, we take advantage of the fact that annihilation in a compound nucleus results in transfer of some of the $1.88 \mathrm{GeV}$ energy to the nucleus by employing high-z stopping materials as seeds in concentrations of up to a few atomic-\%. The resulting nuclear fragments are highly ionizing with short ranges, thus permitting local deposition of a fraction of the annihilation energy.

I In neutron-induced fission, a neutron is added to a target nucleus of mass number A. Fission of the A+1 nucleus occurs when the kinetic energy of the incident neutron plus its binding energy is greater than the critical energy, $\mathrm{E}_{\text {crit }}$ required for this $\mathrm{A}+1$ nucleus to penetrate its internal Coulomb barrier so that the two fission fragments can separate. In fissile nuclei (e.g., $\left.{ }^{235} \mathrm{U}\right)$, the binding energy $\mathrm{E}_{\mathrm{b}}$ released by the neutron in forming the compound $\mathrm{A}+1$ nucleus (e.g., ${ }^{236} \mathrm{U}$ ) is greater than $\mathrm{E}_{\text {crit }}$ so the compound nucleus can fission with zero neutron kinetic energy and such target nuclei have high thermal fission cross sections. In other nuclei (e.g., ${ }^{238} \mathrm{U},{ }^{232} \mathrm{Th}$ ), $\mathrm{E}_{\mathrm{b}}$ is slightly less than $\mathrm{E}_{\text {crit }}$ by an $\mathrm{MeV}$ or so and the balance must be supplied by the kinetic energy of the incident neutron. An example of this is ${ }^{238} \mathrm{U}$ which undergoes only fast fission, with a threshold of $\sim 1 \mathrm{MeV}$ required for the incident neutron. For heavy nuclides in the range ${ }^{233} \mathrm{Bi}$ down to ${ }^{184} \mathrm{~W}$ the formalism from Foderaro [51] indicates that critical energies $\mathrm{E}_{\text {crit }}$ of a minimum of $\sim 10 \mathrm{MeV}$ to several 10's of $\mathrm{MeV}$, respectively, must be supplied to promote nuclear breakup, otherwise de-excitation by gamma emission is the preferred route. Such critical energies can be supplied in antiproton annihilation by the inward-directed pion products with any excess going to the kinetic energy of the nuclear fragments. 


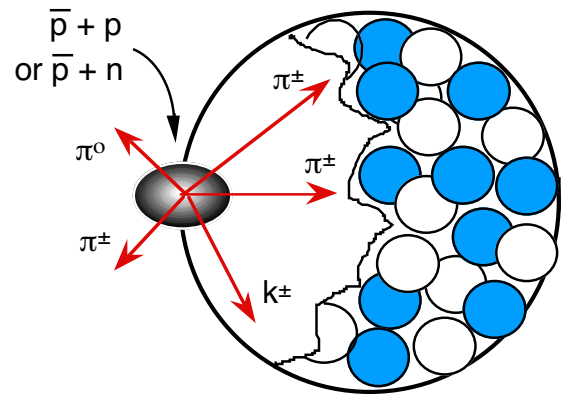

Antiproton annihilates with a proton or neutron on the surface of the heavy nucleus. A fraction of the annihilation pions are directed inwards and interact within the high density nuclear material

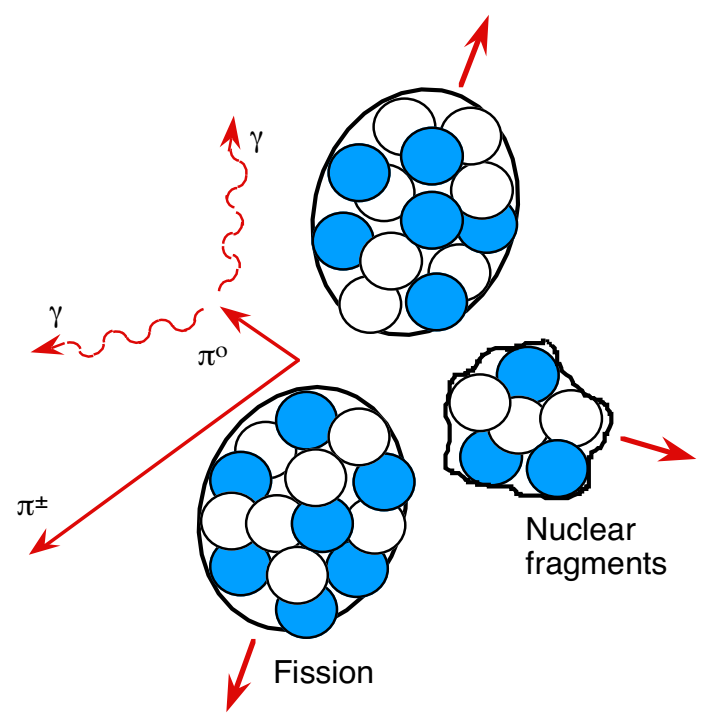

The inward-directed pions cause nuclear breakup resulting in highly ionizing, short range fragments. The outward-directed primary pions and daughters have long ranges and deposit the majority of their energy outside the fusion fuel

Fig. 2. Annihilation of an antiproton in a heavy nucleus takes place with a neutron or proton at the nuclear surface. This results in nuclear breakup with significant energy transfer to the residual nuclear fragments which are highly ionizingwith short deposition ranges.

\section{TARGET CONFIGURATIONS}

\subsection{Inertial Confinement Physics}

High energy gains can be obtained from an ICF capsule if the fusion fuel is heated above the ignition temperature and confined long enough so that an appreciable portion of the fuel is consumed. In inertial confinement, the fusion burn of an ignited fuel mass is limited by hydrodynamic expansion. The fraction of fuel that undergoes thermonuclear burn depends on the fusion reaction rate that scales as the square of the fuel density relative to the fuel disassembly time that scales as the radius of the fuel. For deuteriumtritium (DT) fuel ignited by a central hotspot (see below), the burn fraction can be written in terms of the areal density of the assembled fuel mass - i.e., the product of fuel density and radius, $\rho R\left(\mathrm{~g} / \mathrm{cm}^{2}\right)$ - as approximately [2]

$$
f_{\text {burn }} \approx \frac{\rho R}{\rho R+6}
$$

Thus, for example, an assembled fuel areal density of $3 \mathrm{~g} / \mathrm{cm}^{2}$ would be required to obtain a burn fraction efficiency of around one third.

The complete burning of a 50:50 mix of DT fuel releases a specific energy of $3.38 \times 10^{14} \mathrm{~J} / \mathrm{kg}$. Given that the mass of a spherical fuel assembly scales as $m \sim \rho R^{3} \sim(\rho R)^{3} / \rho^{2}$ we note that, at normal cryogenic solid densities of $\sim 0.25 \mathrm{~g} / \mathrm{cm}^{3}$, a DT fuel mass of some $1.8 \mathrm{~kg}$ would be required to meet the $\rho R=3 \mathrm{~g} / \mathrm{cm}^{2}$ criterion for $33 \%$ 
burnup. This would result in a fusion yield of $\sim 2 \times 10^{14} \mathrm{~J}$; that is, some 50kilotons of highexplosive-equivalent energy release. However, initial compression of the fuel to densities of, say, $200 \mathrm{~g} / \mathrm{cm}^{3}$ at the same $\rho R=3 \mathrm{~g} / \mathrm{cm}^{2}$ condition would reduce this unmanageable yield to $\sim 300 \mathrm{MJ}$ in a fuel mass of only $\sim 3 \mathrm{mg}$, thus making it suitable for terrestrial power production or space propulsion at a repetition rate of several hertz.

A typical ICF fusion target consists of several milligrams of cryogenic solid DT fuel in the form of a spherical shell surrounding a region of low density $\left(\sim \mathrm{mg} / \mathrm{cm}^{3}\right)$ DT gas. The fuel is surrounded by an outer ablator region of mass comparable to that of the fuel and of a material selected to optimize the implosion dynamics. In conventional ICF, energy is rapidly coupled to the ablator from a driver - either directly in the form of symmetrical laser beams or indirectly in the form of x-rays stimulated by lasers, heavyion beams or z-pinches in a surrounding hohlraum [1-6,12,52]. As the heated ablator expands outwards, momentum conservation causes the remaining target to be imploded inward by the rocket effect. With a suitable temporal profile on the drive intensity, the fuel can be maintained relatively cold near the minimum Fermi temperature as it is compressed by the resulting shock waves. At peak drive pressure, the capsule approaches a state of uniform acceleration [11] until spherical convergence effects and gas backpressure cause the fuel to stagnate at high density. As described below, the peak velocity achieved during the implosion is a crucial parameter in determining both the peak compression density and the minimum energy required to cause the fuel to ignite.

Below, we define three major classes of ICF targets, first from a conventional perspective and then describe how they might be adapted for use with antiproton drive. They are differentiated by (a) the target design, (b) how the implosion is performed and (c) whether a separate ignition source is employed .

\subsection{Antiproton Volumetric Ignition}

In volumetric ignition, the bulk of the compressed fuel mass is raised to the ignition temperature, typically $T_{i g n} \gtrsim 3 \mathrm{keV}$ for DT. These types of conditions can be generated in conventional ICF targets if, for example, electrons generated by a highintensity laser interact in a thin $(\sim$ few $\mu \mathrm{m})$ metal or glass shell enclosing a cryogenic solid or liquid DT core. The shell heats rapidly to $\mathrm{keV}$ temperatures, exploding the shell with a pressure of many gigabars and driving an inward shock at a velocity of a $\sim$ few $\times 10^{7} \mathrm{~cm} / \mathrm{s}$. The convergent shock from the shell heats the DT core yielding ion temperatures in the $\sim 3-10 \mathrm{keV}$ range. Such "exploding pusher" targets were the most common types of targets utilized in the early stages of the ICF program and were the first type to produce thermonuclear neutrons. They can more easily achieve higher implosion velocities than the conventional hot-spot targets described below and, due to their low convergences, are more tolerant of asymmetries in the drive. However, they typically do not scale to high gain because the bulk of the DT fuel is preheated. This sets the target mass on a high isentrope which precludes high compression densities with moderate incident energies.

In principle, antiprotons can be arranged to annihilate in the bulk of a compressed DT fuel mass to drive volumetric ignition. However, because of the long range of the annihilation pions produced in the low-z fuel (Fig. 1) the energy deposition efficiency would be low. To ameliorate this problem, seeding the fuel with a small atomic fraction of 
heavy metal can augment energy deposition in the fuel as described above (Fig. 2) but at the expense of high-z impurity mix and attendant increases in radiation losses. Several authors have suggested using antiproton-induced fission to boost the burning of fusion fuel [33-38] and Smith et al proposed the ICAN concept for application to propulsion [34].

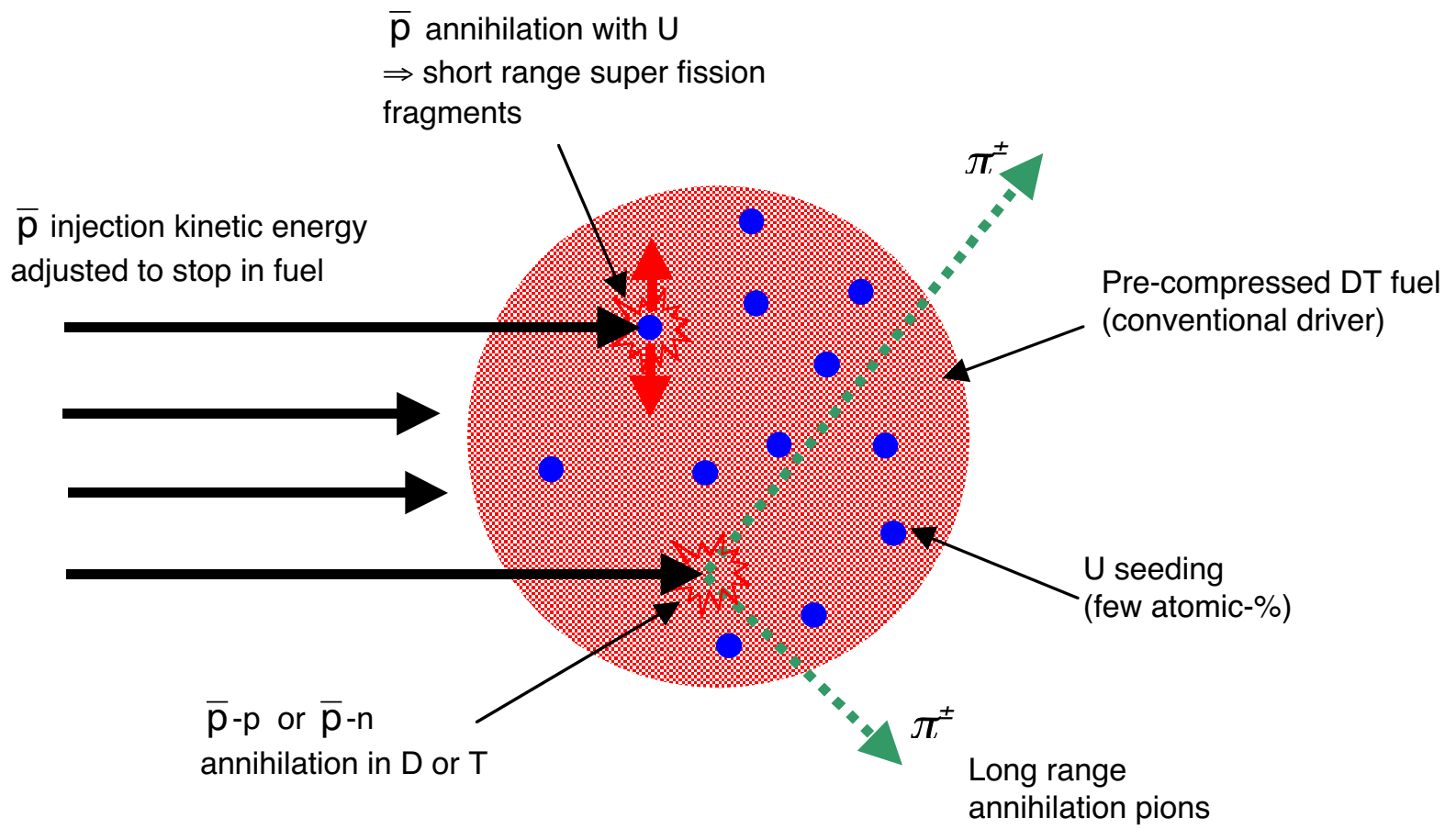

Fig. 3. Antiproton-driven volumetric ignition. Antiprotons are injected into the precompressed fuel which is seeded with heavy metal to enhance energy deposition of annihilation products.

A schematic is shown in Fig 3. Antiprotons annihilate in a DT fuel mass seeded with a heavy metal, say uranium, at the $\sim 0.5-10$ atomic-\% level. The fuel plus seed is precompressed by either by a conventional driver - Smith [34] advocated high intensity, lithium light ion beams - or prior $\overline{\mathrm{p}}$ ablative drive in an outer ablator as described below. Antiprotons preferentially annihilate with the heavy metal seed resulting in energy deposition in the fuel by short range nuclear fragments. The total nuclear yield in the target is the sum of the deposited antiproton induced energy plus the yields from DT fusion and, where applicable, conventional uranium fission induced by fusion neutrons.

\subsection{Antiproton Hotspot Ignition}

The minimum energy required to volumetrically heat a DT fuel mass to ignition temperature $T=T_{i} \sim T_{e}$ is $1.15 \times 10^{11} T(\mathrm{keV}) \mathrm{J} / \mathrm{kg}$, whereas the specific energy to compress it to Fermi-degenerate density $\rho$ is only $\sim 3 \times 10^{8} \rho^{2 / 3} \mathrm{~J} / \mathrm{kg}$, with $\rho$ in units of $\mathrm{g} / \mathrm{cm}^{3}$ [2]. So if the target can be isentropically compressed on a low adiabat - that is, kept relatively cold with minimum temperatures set by the Fermi energy - but ignited from a central high temperature core, gains can be significantly higher and driver sizes reduced accordingly. In such "hotspot" ignition targets, the final assembled state consists of a central, low density, high temperature region containing only a few percent of the fuel mass in approximate pressure equilibrium with the bulk of the surrounding cold compressed fuel 
at high density. The conditions for ignition, i.e., burn propagation from the hotspot into the cold fuel, is approximately a hotspot temperature of $\mathrm{T}_{\mathrm{ign}} \approx 10 \mathrm{keV}$ over a region of local areal density of $(\rho R)_{i g n} \sim 0.3 \mathrm{~g} / \mathrm{cm}^{2}$. The overall fuel burnup and fusion yield is then determined by the total $\rho R$ of the assembled fuel as described above (see Eqn. (1))

In principle, antiprotons could be used to drive a hotspot ignition target replacing the conventional direct or indirect driver system, i.e., laser, heavy-ion beam or z-pinch see Fig 4 where a section of the spherical target is shown. Target dimensions will be discussed in Section 5 below. The kinetic energy of the antiproton beam is controlled so that they annihilate at the ablation front. In the target designs of this paper, the kinetic energy required of the injected antiprotons is typically less than one percent of their net deposited annihilation energy. As with volumetric ignition above, a heavy metal seed will be required to enhance antiproton energy deposition. Here, however, the seed is placed only in the ablator and not in the fuel itself.

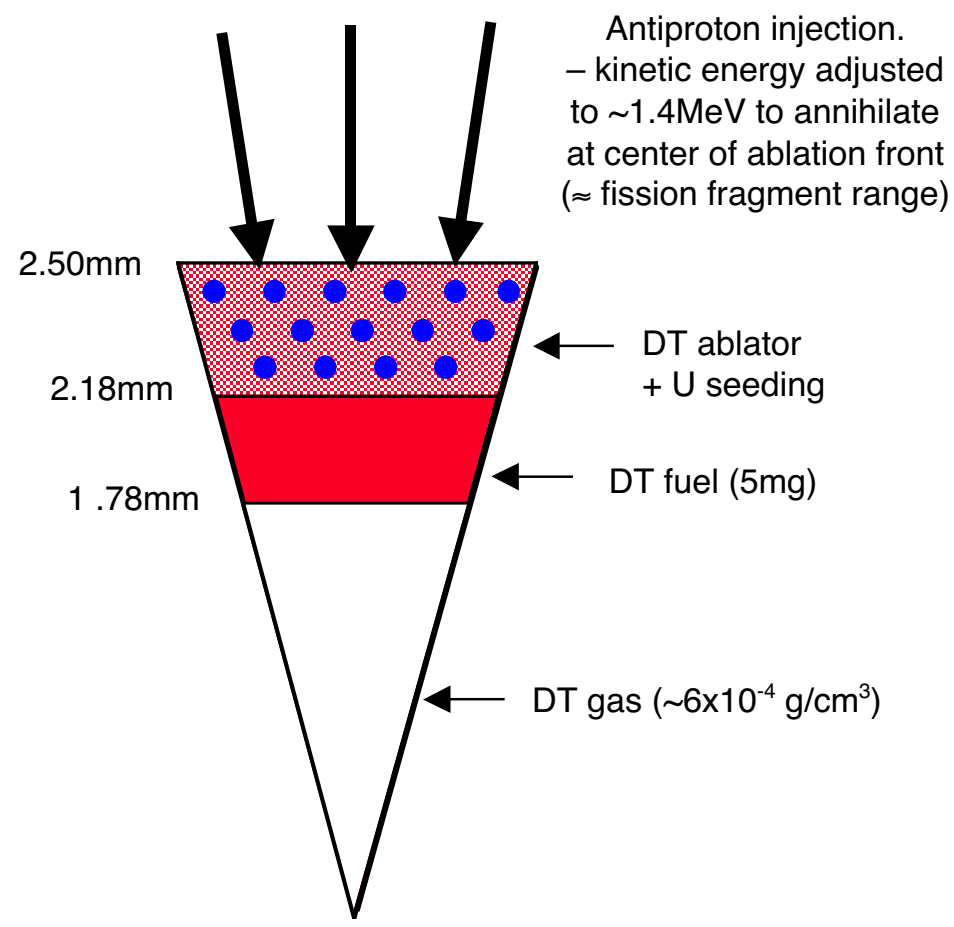

Fig. 4. Section of the spherical target for antiproton-driven hotspot ignition. A direct drive configuration is shown here but semi-indirect drive options are also possible. Target specifications are discussed in Section 5.

In the hotspot ignition example analyzed below, we will focus on a direct-drive concept where the antiprotons annihilate directly with the ablator shell although the principles could be applied to indirect-drive via antiproton-induced $x$-ray production within a high-z hohlraum case. A "semi-indirect-drive" antiproton-driven configuration may also be possible, where the ablator is surrounded by a thin outer high-z shell stood off from the ablator by a foam buffer layer. The kinetic energy of the antiprotons would be adjusted to penetrate the shell and interact in the ablator with the resulting radiation contained by the shell acting as a close-coupled spherical hohlraum.

Relative to $\overline{\mathrm{p}}$-driven volumetric ignition, we would expect to realize the following advantages with $\overline{\mathrm{p}}$-driven hotspot ignition: 
- Clean DT fuel - the heavy metal only seeds the ablator so that the fuel remains at $\mathrm{Z}_{\mathrm{eff}} \sim 1$

- Higher gains - only a small fraction of the fuel is initially heated to ignition temperatures; therefore, less incident antiprotons are required per target

- Requirements on antiproton timing and spatial precision are relaxed allowing longer injection times and larger focal spots. However temporal pulse shaping of the $\bar{p}$ beam will be required to maintain the compressed fuel on a low adiabat

Relative to conventional direct drive ICF, other than the obvious advantage of a profound reduction in driver system mass, antiprotons should be capable of producing higher rocket efficiencies - that is a greater maximum kinetic energy of the imploding shell relative to the initial energy expended in outward ablation. A conventional direct drive target has a rocket efficiency of only $\sim 5-10 \%$, several times less than the ideal efficiency, because the exhaust is continually heated by the incident laser flux driving the ablation. By contrast, the kinetic energy of the antiprotons is adjusted so that they deposit their annihilation energy in the unablated shell with negligible energy lost in transmission through the exhaust stream. Also, given that the typical annihilation pion range in DT is greater than $100 \mathrm{~g} / \mathrm{cm}^{2}$ (see below), preheating of the fuel during compression by inward directed pions is probably not a problem because areal fuel densities are $<1 \mathrm{~g} / \mathrm{cm}^{2}$. Finally, as we shall see below, although operated in a direct drive mode, the characteristics of antiproton energy deposition by fission fragments result in ablation fronts and ablation velocities more akin to those encountered in conventional indirect-drive targets with radiation ablation. Accordingly, the enhanced stability features of indirect-drive ICF may also pertain.

\subsection{Antiproton Fast Ignition}

The compression energy required for hotspot ignition above could be reduced, and the target gain further increased, if the fuel is initially compressed to a density greater than a typical density in the hotspot but less than the cold bulk fuel density and then separately ignited over a small region. This is the principle of "fast" ignition where the compression of a target is decoupled from its ignition [10,53]. First, a slow ( 50-100 ns) driver is employed to compress the fuel to intermediate densities. Then, the ignition energy is delivered rapidly by a separate, fast ( tens of picoseconds) system. In conventional fast ignition scenarios, this energy is supplied by a low energy, high intensity, short-pulse laser and transferred to the high density region by the transport of suprathermal electrons or focused ions [54]. The hotspot so formed is on the outside of the compressed fuel and is at the same density as the main fuel mass. Because hotspot and fuel are not in pressure equilibrium, the ignition criteria now dictate $T_{\text {ign }} \gtrsim 10 \mathrm{keV}$ over a local areal density of $(\rho R)_{i g n} \sim 0.5 \mathrm{~g} / \mathrm{cm}^{2}$ for propagating burn, up from the $(\rho R)_{i g n} \sim 0.3 \mathrm{~g} / \mathrm{cm}^{2}$ condition for hotspot ignition. However, because less compression energy was initially expended, larger gains are predicted for the same fuel mass.

As shown in Fig. 5, antiprotons could be used in principle to accomplish fast ignition, either as the ablative drive system to compress the fuel in a manner similar to hotspot ignition above but with relaxed timing constraints, and/or as a separate fast energy source to spark the burn. As with conventional fast ignition concepts, a reentrant 
cone could be employed to shield the ignition region from the compression blowoff debris.

A speculative alternative to injecting antiprotons as the fast ignition energy source might be to pre-emplace them at the center of the capsule and isolated by a magnetic field before the slow drive occurs. The central inner capsule is then squeezed down by the main compression to trigger the antiproton release. We note that conventional ICF capsules with pre-emplaced magnetic fields have been previously studied [55-57]

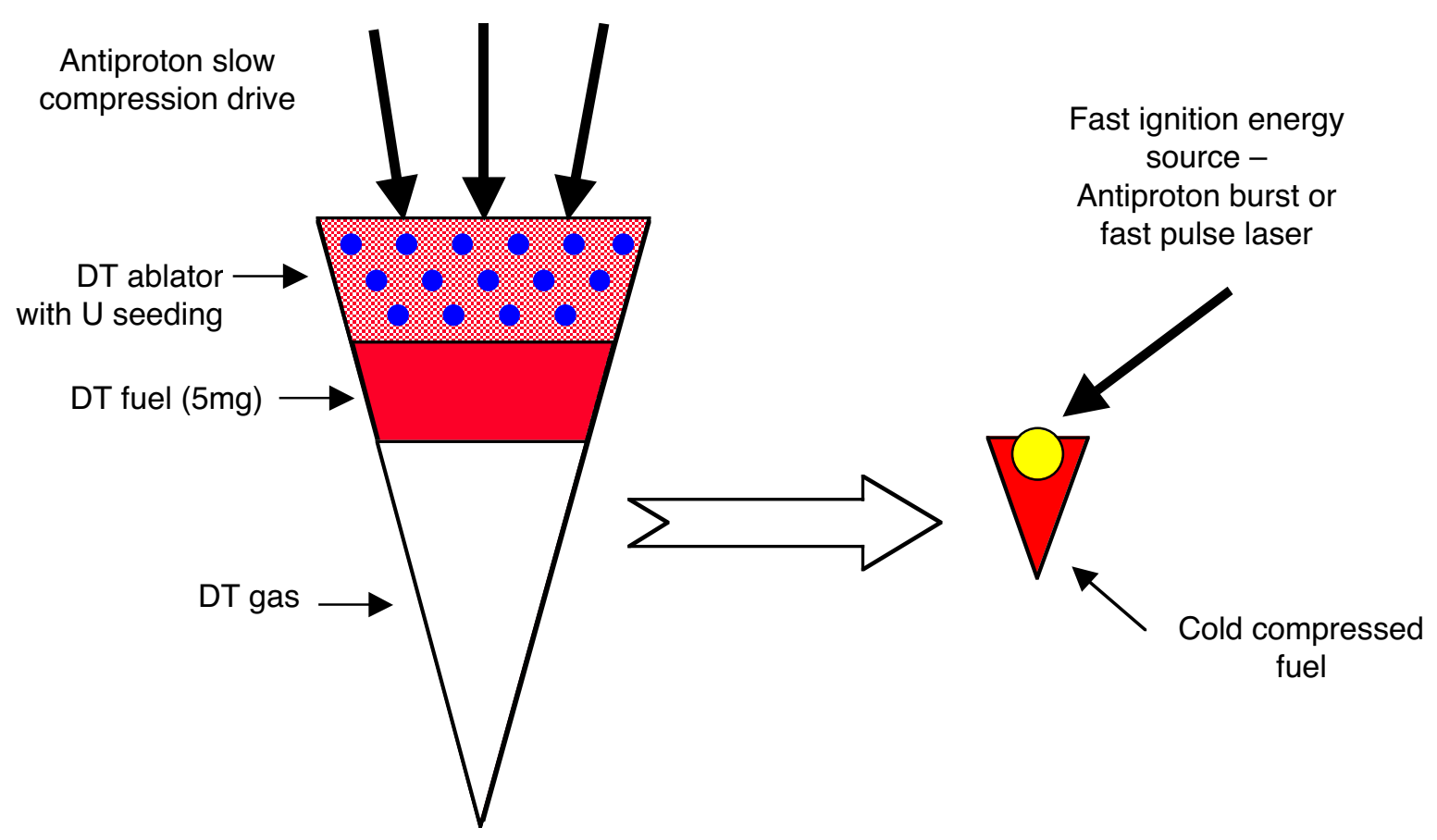

Fig. 5. Section of the spherical target for antiproton-driven fast ignition.

Both antiproton-driven hotspot ignition (Fig. 4) and fast ignition (Fig. 5) require the formation of a local ignition hotspot in the compressed fuel. The distinguishing parameter of these two schemes during the compression phase is the required velocity at which the fuel is assembled, this being relaxed for fast ignition with commensurate relaxation of the antiproton injection time. In fact, hotspot ignition might be better differentiated from fast ignition by calling it "fast compression ignition". The targets for both antiproton hotspot ignition and fast ignition would be similar - i.e., an inner unseeded layer of DT fuel surrounded by an outer seeded ablator. However, as shown below, because of the relaxed timing constraints, fast ignition capsules could be designed with a thicker, more massive shell that is more tolerant of instability growth.

Of the various methods of $\bar{p}$-driven ICF, using antiprotons as the energy spark for the fast ignition hotspot presents the most stringent requirements in terms of injection times and aiming precision. Accordingly, it seems expedient to focus on the utility of antiprotons to drive the relaxed, slow compression phase of fast ignition, while assuming a conventional fast energy source such as a short pulse laser is used to spark the ignition. 


\subsection{Muon Catalysis}

We note from Fig. 1 that negative muons are produced in the decay of the annihilation pions. Deposition of these muons in DT fuel could, in principle, promote fusion by muon catalysis. Here, a thermalized heavy negative muon binds tightly to a fuel nucleus and reduces the width of the conventional repulsive Coulomb barrier between it and a neighboring fuel nucleus by a factor of $m_{u} / m_{e} \sim 200$, thus permitting fusion to proceed at significantly below eV temperatures [58]. Typically, a maximum of $\sim 150 \mathrm{DT}$ fusions can be catalyzed per muon because the latter is ultimately lost by sticking to an outgoing alpha particle. This could result in an additional energy deposition of up to $\sim 500 \mathrm{MeV}$ per original $\overline{\mathrm{p}}$ via $3.5 \mathrm{MeV}$ fusion alpha particles. However, only a small fraction of the muons produced by pion decay will be captured locally. Also, the lifetime for $\pi^{-}$decay to muons is $\sim 70 \mathrm{~ns}$ in the lab frame which is too slow for muon catalysis to contribute usefully to energy deposition in any of the above target designs other than, perhaps, the slow compression phase of fast ignition. Furthermore, ultimate muon decay ( $\sim 6 \mu \mathrm{s}$ in the lab frame) is too fast to permit muon collection and re-injection into a subsequent capsule unless the latter is a separate component of the same target assembly. Accordingly, we will not consider muon catalysis further in the antiproton energy deposition process.

\section{ANTIPROTON-DRIVEN FISSION-FUSION VOLUMETRIC IGNITION}

\subsection{Analysis Method}

The target for this concept was introduced in Section 3.2 above and shown in Fig. 3. We will here consider antiprotons to annihilate in a precompressed DT fuel mass seeded with a heavy metal. In this example we will use uranium in the form of ${ }^{238} \mathrm{U}$ as the seed - this closely approximates natural uranium at $99.3 \%{ }^{238} \mathrm{U}$ - although other heavy metals such as tungsten through bismuth could be employed. The advantage of uranium is that it can also undergo conventional fast fission so that additional target yield can result from subsequent fusion-neutron-induced fission in the seed material. We assume that fuel precompression is performed by a conventional driver; in the next section we will examine fuel compression by antiproton-driven ablation. Antiprotons preferentially interact with the metal seed resulting in energy deposition in the fuel mainly by short range nuclear fragments rather than the long range pions that would result from annihilation in pure DT. The total energy produced in the target is the sum of the DT fusion yield and subsequent fusion-neutron-induced uranium fission yield.

We proceed with the analysis as follows, employing 1-D simulations with the LASNEX radiation-hydrodynamics-burn code [59]. We start a given DT fuel mass at $t=0$ at full compressed density and a given uranium seed fraction. We investigate the fuel ignition/burn conditions and overall yield as a function of the initial temperature of the compressed fuel. We then compute the requirements for antiproton slowing down, capture and energy deposition that would produce a volumetric ignition energy source consistent with these initial conditions and thereby deduce the required number of injected antiprotons together with the necessary initial compression energy. There are thus four independent variables for this analysis: DT fuel mass, ${ }^{238} \mathrm{U}$ seed fraction, initial density (i.e., initial $\rho-\mathrm{R}$ ) and initial temperature. 
We assume the initial kinetic energy of the antiproton beam is adjusted to annihilate within the bulk of the seeded DT fuel. As the nuclear fragment range is much less than the compressed fuel radius, we take the fragment energy to be deposited where born. (In Section 5 below on hotspot ignition targets with antiproton-driven ablation, we will need to accommodate fission fragment ranges explicitly). By contrast, the annihilation pion range is typically greater than the fuel dimensions and we compute its fractional energy deposition consistently. To avoid computing time-dependent slowing down, we assume that the ignition energy is deposited in a time less than the hydrodynamic disassembly time of the compressed fuel, thus setting a maximum pulse length on the injected antiprotons; this parameter increases (i.e., becomes less stringent) as the seed fraction and target mass increase.

We might expect that we need sufficient uranium seed fraction to preferentially capture the antiprotons and promote enhanced local energy deposition but not too high otherwise unacceptable "mix" will result with high $\mathrm{z}_{\text {eff }}$ and deleterious Bremsstrahlung losses. Therefore, we might expect there is an optimum seed fraction that will maximize the target gain - that is, maximize the ratio of nuclear yield to the number of injected antiprotons required to establish the initial ignition conditions. In fact, as we shall see, an increasing $\mathrm{U}$ seed fraction results in a greater fraction of the yield coming from conventional fast neutron fission in ${ }^{238} \mathrm{U}$ relative to DT fusion and there is no clear optimum in the burn energetics.

\subsection{Burn Calculations}

We select an initial DT mass of 5mg for these example calculations, typical of a fuel mass used in conventional high yield ICF reactor applications [3-6], and assume that it is precompressed by a conventional driver to an areal density of $\rho R=3 \mathrm{~g} / \mathrm{cm}^{2}$, that is, an average density of $\rho \sim 150 \mathrm{~g} / \mathrm{cm}^{3}$ (for unseeded pure DT) and spherical radius of $\sim 0.02 \mathrm{~cm}$. The compression energy will be considered further below. If this compressed mass was ignited by a central hotspot we would expect, by Eqn. 1, a fuel burnup of around onethird and a fusion yield of $\sim 560 \mathrm{MJ}$. As we shall see, volumetric ignition results in a lower achievable yield. Employing LASNEX in 1-D spherical geometry, we determine the minimum volumetric ion temperature at which the fuel ignites - that is, produces a significant multiplicity in ion temperature together with a substantial fusion yield. The antiproton deposition characteristics necessary to achieve these initial conditions will be considered below. The dashed line in Fig. 6 shows the yield for pure unseeded DT as a function of the initial volumetric ion temperature $T_{o}$. The required initial temperature for ignition is seen to be around $T_{i g n}=T_{o} \sim 2.5-3 \mathrm{keV}$ where a yield of $\sim 300 \mathrm{MJ}$ is achieved. The yield increases with increasing $T_{o}$ above $3 \mathrm{keV}$ and saturates at $\sim 420 \mathrm{MJ}$ around $T_{o}$ $\sim 10-20 \mathrm{keV}$. Once the initial temperature is at or above the ignition temperature, peak ion temperatures climb during the burn to $\sim 100 \mathrm{keV}$. We note these yields are typically less than those achievable with central hotspot ignition under the same $\rho$ - $R$ conditions because the fuel is volumetrically preheated and outward expansion quenches the burn more rapidly. 


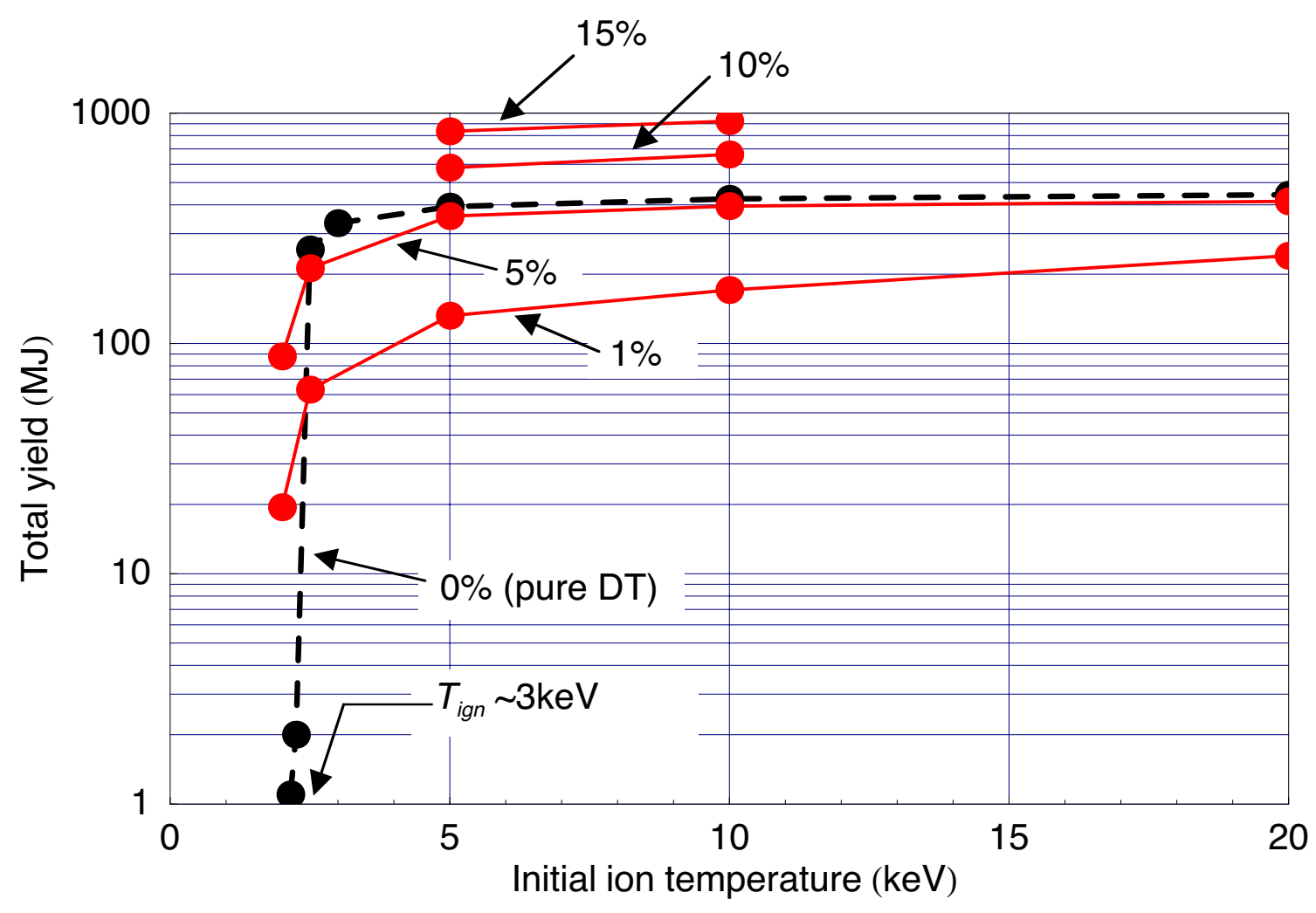

Fig 6. Total yield (fusion plus fission) versus initial fuel temperature for various uranium seed fractions under volumetric ignition with a $5 \mathrm{mg}$ DT fuel mass. The dash line is for pure, unseeded DT

Although a fusion yield of $420 \mathrm{MJ}$ rep-rated at, say, $10 \mathrm{~Hz}$ would give a respectable $4.2 \mathrm{GW}$ average power, a large number of antiprotons would be required to drive each target due to the minimal energy deposition of annihilation pions in pure DT. Accordingly, we now determine the target burn conditions as a function of uranium atomic seed fraction $f_{U}$ defined as

$$
f_{U}=\frac{n_{u}}{n_{u}+n_{H}}
$$

where $n_{U}$ and $n_{H}$ are the number densities of ${ }^{238} \mathrm{U}$ and $\mathrm{D}+\mathrm{T}$, respectively. We maintain the DT fuel mass and initial radius at the values above $(5 \mathrm{mg}$ and $0.02 \mathrm{~cm}$, respectively). Therefore, increasing the uranium seed fraction $f_{U}$ increases the initial mass density and areal density as $150+\left(1.42 \times 10^{4} f_{U}\right) /\left(1-f_{U}\right) \mathrm{g} / \mathrm{cm}^{3}$ and $3+\left(283 f_{U}\right) /\left(1-f_{U}\right) \mathrm{g} / \mathrm{cm}^{2}$, respectively.

The result of seeding the DT fuel is shown in Fig. 6, where yields are plotted versus initial ion temperature for uranium seed fractions $f_{U}$ in the range zero to $15 \%$ atomic. Given that a uranium atom is a hundred times heavier than the average "DT atom", the uranium mass exceeds the DT mass above $\sim 1 \%$-atomic seed fraction. The yields for low seed fraction targets $\left(f_{U}<5 \%\right)$ are less than pure DT because the heavy metal seeding increases the radiation losses during burn and peak ion temperatures are suppressed. However, at seed fractions above 5\%, yields are maintained in the several hundred megajoule range and actually increase for the higher seeded targets. This is 
because primary and down scattered $14 \mathrm{MeV}$ neutrons from the DT fusion reaction can promote fast fission in the uranium seed nuclei within the compressed fuel (the neutron threshold energy is $\sim 1 \mathrm{MeV}$ ). Our LASNEX calculations evaluate the neutron transport and fission rates consistently with the fusion burn so that as $f_{U}$ increases, an increasing fraction of the total yield shown in Fig. 6 is due to conventional ${ }^{238} \mathrm{U}$ fast fission. The partitioning of the fission and fusion yields will be discussed below, where we show that the fission yield becomes the dominant contributor to the total yield above a seed fraction of about $13 \%$. The seeded targets have considerably lower peak burn temperatures relative to pure DT but comparable yields. For example, pure DT with $f_{U}=0$ demonstrated a maximum yield of $\sim 420 \mathrm{MJ}$ with peak ion temperatures of $\sim 100 \mathrm{keV}$. By contrast, a target with $f_{U}=5 \%$ exhibits a peak burn temperature of only $\sim 25 \mathrm{keV}$ but comparable peak yields of $\sim 400 \mathrm{MJ}$. Thus the increasing $\mathrm{z}_{\mathrm{eff}}$ and radiation losses with increasing seed fraction that suppress peak burn temperatures are offset by the increasing fission yield and by the tamping effect of the larger target mass. For example, the disassembly time for a $1 /$ e reduction in the initial density is a factor of three longer for the $f_{U}=5 \%$ target relative to the pure DT target.

\subsection{Antiproton Energy Deposition.}

Given the burn dynamics above, we now estimate the antiproton injection requirements to initiate the target conditions at $t=0$. As shown in Fig 2, annihilation with a heavy nucleus takes place with a proton or neutron near the surface. The outward directed pions or kaons have long ranges even in the pre-compressed fuel (see below) so only a small fraction of their ionization energy will be deposited . Similarly, the neutral pion decays in $\sim 10^{-18}$ s into two energetic gamma rays which also leave the target with minimal local energy deposition. By contrast, the mesons directed into the heavy nucleus encounter a medium of nuclear density. Their interaction mean free path is then approximately

$$
\lambda=[n \sigma]^{-1} \approx \frac{\frac{4}{3} \pi r_{N}^{3}}{A \sigma}
$$

where $n$ is the nucleon number density, $\sigma$ is the pion-nucleon interaction cross section, $A$ is the nuclear mass number and $r_{N}$ is the nuclear radius. Taking $\sigma \sim 100 \mathrm{mb}$ for $\sim 100 \mathrm{MeV}$ pions [60] and $r_{N}=1.2 A^{1 / 3} \times 10^{-15} \mathrm{~m}$, gives a nuclear density of $n \sim 1.4 \times 10^{44} \mathrm{~m}^{-3}$ for $\mathrm{A}=238$ and, thus, a pion mean free path of only $\sim 7 \times 10^{-16} \mathrm{~m}$. This is about a tenth of the nuclear radius thus ensuring that all the inward directed pion energy is transferred to the nucleus and its subsequent breakup. The resulting nuclear products are highly ionizing with short ranges.

An antiproton slowing down in the seeded fuel will be captured by either a $U$ or $\mathrm{D} / \mathrm{T}$ nucleus when its local velocity has reduced to the order of that of an outer (bound) orbital electron in that nucleus. For DT (i.e., hydrogen), the radius of the first electron Bohr orbit is $\sim 5.3 \times 10^{-11} \mathrm{~m}$ and its orbital velocity is $v_{0}=\alpha c=2.2 \times 10^{6} \mathrm{~m} / \mathrm{s}$, where $\alpha$ is the fine structure constant. The equivalent kinetic energy of an antiproton with this velocity is $\sim 25 \mathrm{keV}$. Assuming that the incident kinetic energy of the antiproton is adjusted so that it always stops in the bulk fuel by annihilation with either ${ }^{238} \mathrm{U}$ or DT, the total fraction of 
the available $1.88 \mathrm{GeV}$ annihilation energy deposited in the fuel therefore depends on the probability of antiproton capture by uranium to the probability of capture by a DT nucleus. Using the conventional method of combining macroscopic cross sections, $\Sigma$, we write the probability of capture by uranium as

$$
p_{U}=1-p_{H} \approx \frac{\Sigma_{U}}{\Sigma_{U}+\Sigma_{H}}=\frac{n_{U} \sigma_{U}}{n_{U} \sigma_{U}+n_{H} \sigma_{H}}
$$

where $n_{U}$ and $n_{H}$ are the number densities of uranium and D+T, respectively, and $\sigma_{U}$ and $\sigma_{H}$ are the microscopic capture cross sections and $p_{H}$ is the probability of capture by DT. Using a semi classical argument, Morgan and Hughes [61] show that the $\overline{\mathrm{p}}-\mathrm{H}$ collision capture cross section can be written

$$
\sigma_{H}=k(c / v) \pi r_{o}^{2}
$$

where $v$ is the relative $\bar{p}-H$ velocity, $r_{o}$ is the classical outer electron radius and $k=3.60 \times 10^{5}$ is a dimensionless constant. Taking $r_{o}$ for DT as the first Bohr orbit and, by analogy, an outer electron radius for ${ }^{238} \mathrm{U}$ as $\sim 1.38 \times 10^{-10} \mathrm{~m}[62]$ we can use Eqn. 4 to estimate the probability of capture by the heavy metal as a function of uranium seed fraction $f_{U}$ defined in Eqn. 2 above. The energy deposited in the fuel per antiproton injected can now be determined as a function of the uranium seed fraction $f_{u}$ as

$$
E_{n e t}\left(f_{u}\right) \approx p_{u}\left(f_{u}\right) p_{f}<E_{f}>+\left(1-p_{u}\left(f_{u}\right)\right) \frac{\rho R\left(f_{u}\right)}{(\rho R)_{\pi}}<E_{\pi}>
$$

where the first and second terms represent the energy deposition contributions from short range uranium breakup and long range DT-produced pions, respectively, and where $p_{u}$ is the probability of capture by uranium, $1-p_{H}$ is the probability of capture by DT, $p_{f}$ is the probability that an annihilation event with an outer ${ }^{238} \mathrm{U}$ nucleon will result in fission of the nucleus, $\left\langle E_{f}\right\rangle$ is the average energy of the super fission fragments, $\rho R$ is the areal density of the compressed fuel, and $(\rho R)_{\pi}$ is the areal range of the charged annihilation pions of average energy $\left\langle E_{\pi}>\right.$.

The effects of applying Eqns. 4 and 6 are shown in Fig 7 where the capture probabilities on uranium and DT are shown as a function of seed fraction $f_{u}$, together with the resulting net energy depositions expressed as a fraction of the gross $1.88 \mathrm{GeV}$ annihilation energy. Theses results use (a) a $\bar{p}$-induced fission probability for uranium of 0.8 which is the average of experimental results from Schmid [30], (b) a total super fission fragment energy release (including the incident pion energies and Coulomb repulsion energy) of $\sim 700 \mathrm{MeV}$ from VonEdigy [29], and (c) a total charged pion kinetic energy of $713.5 \mathrm{MeV}$ distributed, on average, over $1.5 \pi^{+}$and $1.5 \pi^{-}$[49]. We estimate the pion range $(\rho R)_{\pi}$ in a mixture of $U$ and DT by noting that it can be written in terms of a proton range in the same material at the same velocity multiplied by the ratio of the pion mass to the proton mass; the method for the proton range calculation is discussed below. 


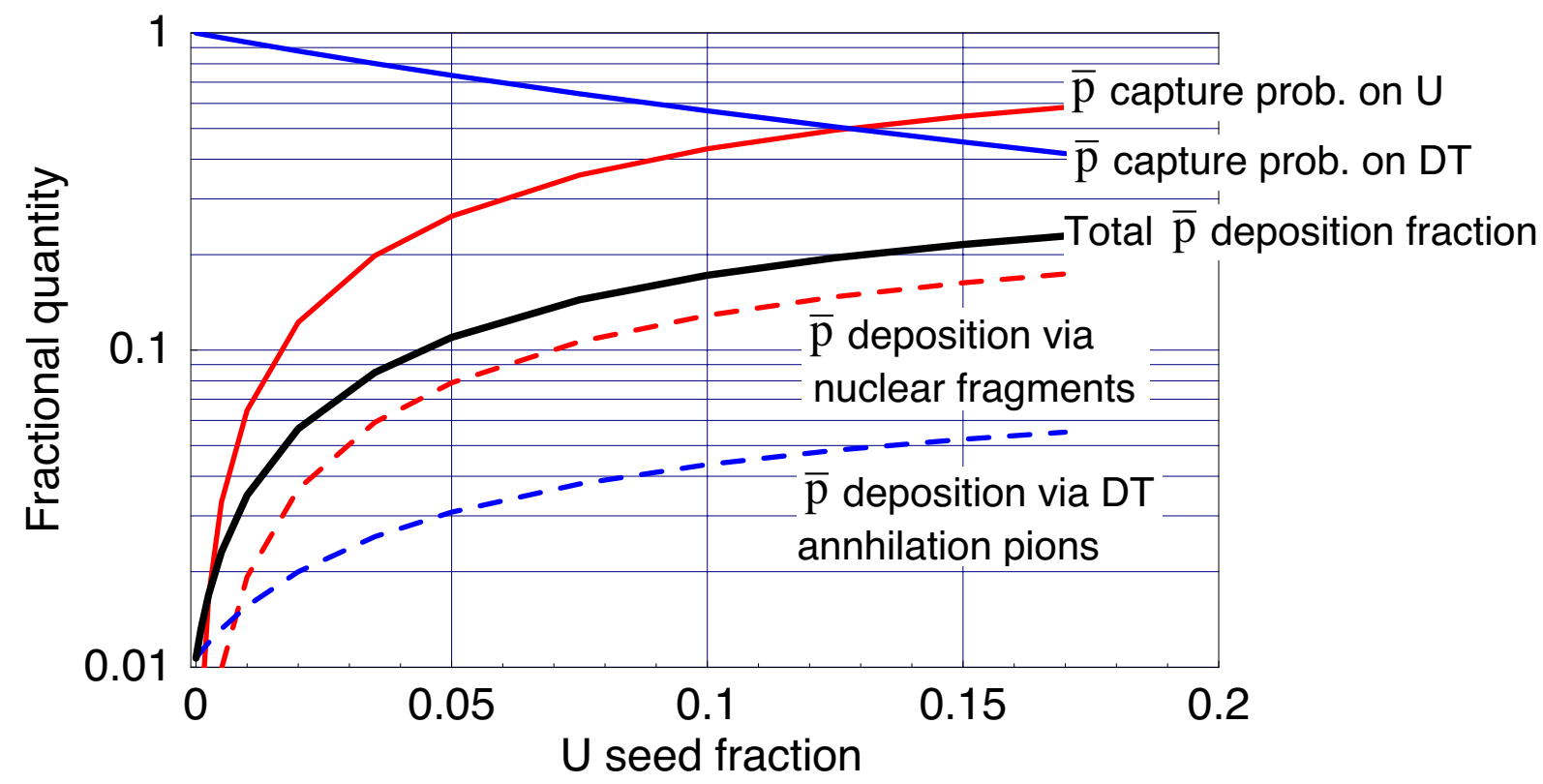

Fig. 7. Energy deposition efficiency of $\overline{\mathrm{p}}$ annihilation products versus uranium seed fraction. Energy deposition by uranium nuclear fragments exceeds that due to DT annihilation pions above a seed fraction of 0.007

In Fig. 7, the fractional capture on uranium rises quickly with increasing $f_{u}$, a consequence of the larger capture cross section of uranium relative to that of DT. A seed fraction of $\sim 13 \%$ is seen to result in equal capture probability on either ${ }^{238} \mathrm{U}$ or DT. Moreover, because of the much larger local energy deposition resulting from uranium capture, a seed fraction of only $f_{u} \sim 0.007$ is required for the energy deposition in the fuel by nuclear fragments to exceed that from pions from DT capture. A 0.01 seed fraction gives a $3.6 \%$ net deposition fraction, or some $70 \mathrm{MeV}$, whereas a seed fraction of 0.05 is required for greater than $10 \%$ of the total annihilation energy - that is, greater than $188 \mathrm{MeV}$ - to deposit in the fuel.

As an independent check on this methodology, we can also estimate capture probability by using inverse stopping ranges as

$$
p_{U}=1-p_{H} \approx \frac{R_{U}^{-1}}{R_{U}^{-1}+R_{H}^{-1}}
$$

where $R_{U}$ and $R_{H}$ are the stopping range in pure uranium and pure hydrogen media, respectively, with individual number densities of $n_{U}$ and $n_{H}$, Ranges are obtained from

$$
R\left(E_{\bar{p}}\right)=\int_{E_{\bar{p}}}^{E_{\bar{p}}} \frac{-d E}{d E / d x}
$$

and where $E_{\bar{p}}$ is the injected kinetic energy of the antiproton and $E_{o}$ is its residual kinetic energy at which its velocity becomes equal to the outer orbital electron of the capturing nucleus. Stopping powers $d E / d x$, for protons on uranium and hydrogen were taken from 
Anderson and Zeigler [63] which are accurate for $1 \mathrm{keV} \leq E_{p} \leq 100 \mathrm{MeV}$. The required injection kinetic energies for antiproton penetration through the compressed, seeded DT are discussed below but we note that because a ratio of ranges is used in Eqn. 7, the precise upper limit is not crucial to evaluate Eqn 8. The result of using Eqn.7 gives capture probabilities that are the same within $5 \%$ or less of those separately evaluated by Eqn. 4 and shown in Fig. 7. For example, for a seed fraction of $f_{u}=2 \%$, Eqn 4 gives a capture probability on uranium of 0.122 while Eqn. 7 evaluates to 0.128

\subsection{Performance Parametrics}

Armed with the energy deposition formalism from Eqn. 6 and the total source energy present at $t=0$ from the LASNEX calculation, we can now infer the required number of antiprotons that would yield this energy source. We assume that the antiproton flux intercepts the seeded core and that their energy is deposited in a time less than the hydrodynamic disassembly time of the compressed fuel; injection timing and aiming precision will be discussed in Section 7 below.

Fig 8 shows the resulting yield curves, where the total (fission plus fusion) yield for the $5 \mathrm{mg}$ (initial unseeded DT mass) target is plotted as a function of the number of injected antiprotons, with increasing uranium seed fractions shown as a parameter. The partition between fusion and fission is shown below. The points along each curve represent increasing initial ion temperature $T_{0}$ as shown. Saturated yields are $\sim 400 \mathrm{MJ}$ for pure DT but then decrease initially for low uranium seeding due to increasing $z_{\text {eff }}$ and Bremsstrahlung losses. The yields then recover at higher seeding fractions because of increased fusion-induced fission contribution.

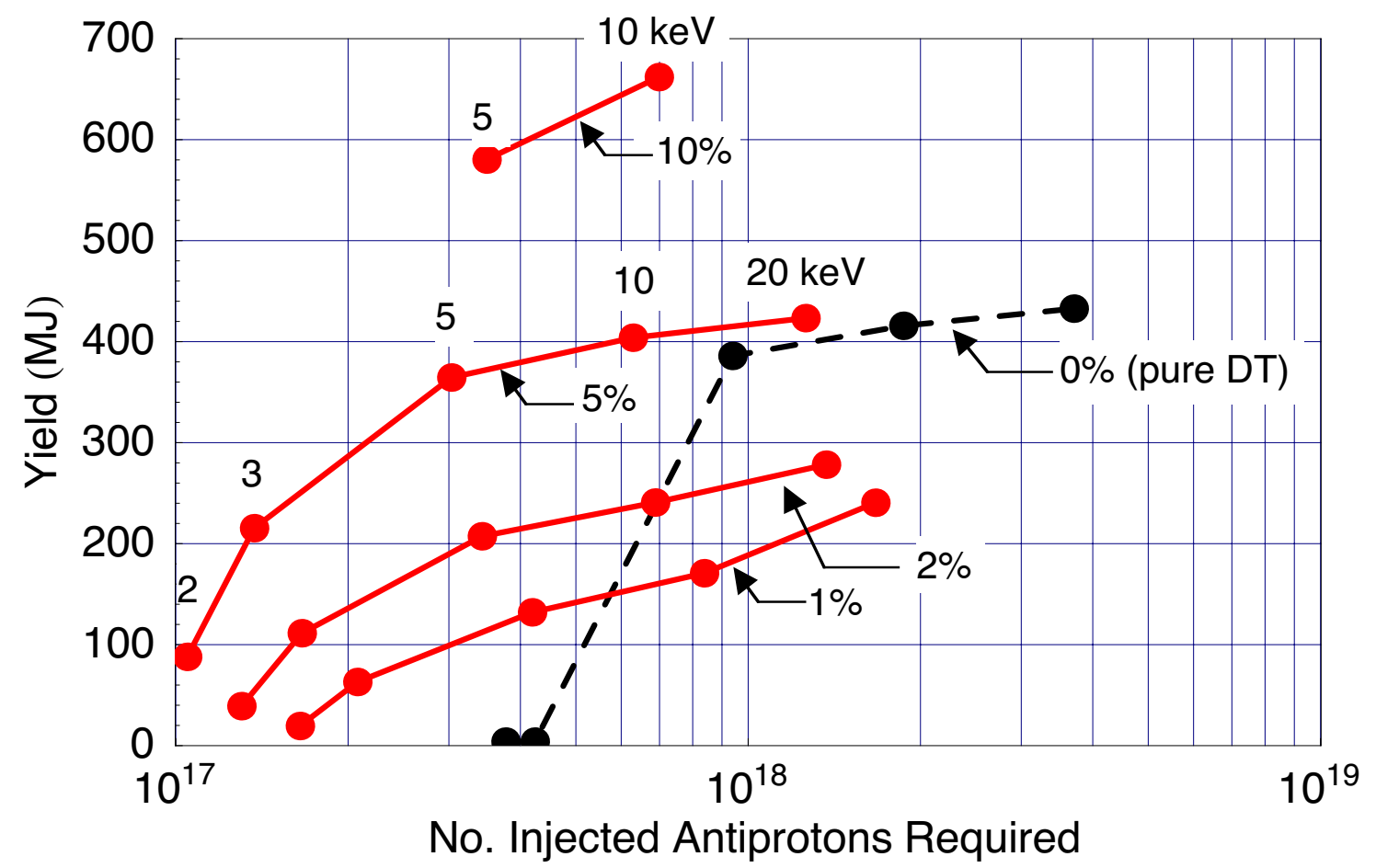

Fig. 8 Volumetric ignition yield curves versus number of injected antiprotons for precompressed fuel. The uranium seed fraction is shown as a parameter. The points along each curve represent increasing initial ion temperature $T_{0}$ in $\mathrm{keV}$ 
Fig 9 shows the corresponding target gain curves where gain is defined as the total fusion plus fission yield divided by the total injected antiproton annihilation energy (i.e., 1.88GeV per injected antiproton). Capsule gains - that is, total fusion plus fission yield divided by the annihilation energy deposited in the fuel-would be some ten to thirty times higher depending on seed fraction. Because yields first climb strongly with increasing ion temperature but begin to roll over around $T_{o} \sim 5-10 \mathrm{keV}$, gains maximize for an injected antiproton burst in the range 1-2 $\times 10^{17}$. That is, except for pure DT which requires an order of magnitude more antiprotons due to the low energy deposition efficiencies; gains are correspondingly low ( unity) for a pure DT target.

Fig 10 shows the partition between fusion and fission yields as the uranium seed fraction is increased. At each seed fraction, yields were selected from the particular LASNEX case run at an initial ion temperature of $T_{o}=10 \mathrm{keV}$ because, from Fig. 6 , yields were tending to saturate around that ion temperature. We note that the fusion (and total) yield initially drops due to increasing Bremsstrahlung losses with increasing seed fraction. For example, $z_{\text {eff }}$ increases from unity at zero seed to 1.44 at $f_{u}=0.5 \%$ and 1.88 at $f_{u}=1 \%$ (all at $T_{o}=10 \mathrm{keV}$ ). Above $f_{u} \sim 1 \%$, the fusion yield begins to increase again as the yield from uranium fission becomes appreciable and the burn temperature is sustained via the $\sim 200 \mathrm{MeV}$ of fission product energy per (conventional) fast fission event. Also, as described above, the increasing heavy metal fraction is contributing to tamping of the target, thus slowing its disassembly during burn. The result is that both fission and fusion yields increase. At a seed fraction of 5\%, the target recovers its initial yield of $400 \mathrm{MJ}$ but now with a quarter coming from fission. At a seed fraction of $\sim 13 \%$ the fission and fusion yields are equal at $\sim 420 \mathrm{MJ}$, for a total yield of $\sim 840 \mathrm{MJ}$.

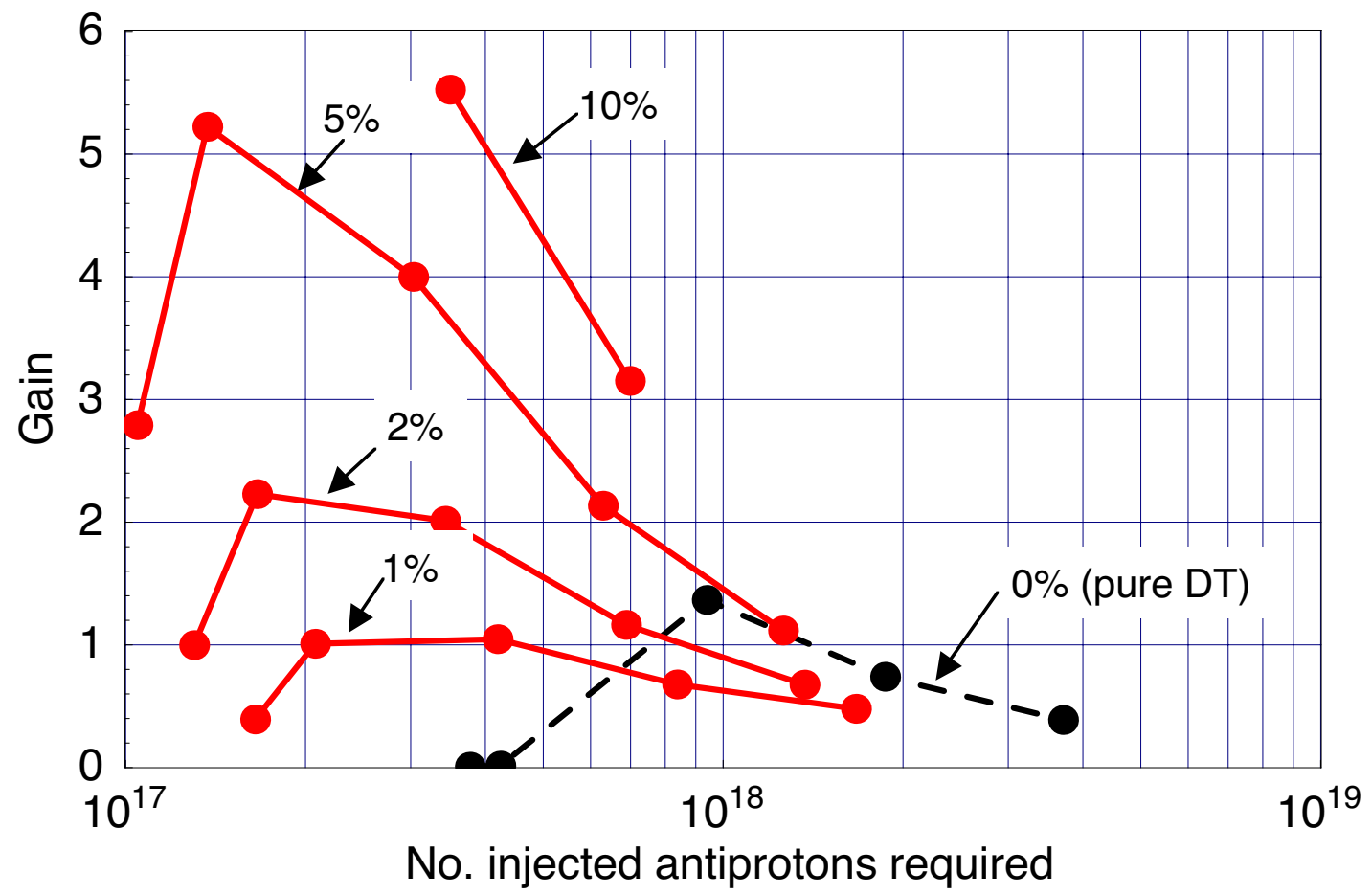

Fig. 9 Volumetric ignition gain curves versus number of injected antiprotons with the uranium seed fraction as a parameter. The points along each curve represent increasing initial ion temperature $T_{0}$ (see Fig. 8). These do not include the initial compression energy required to precompress the fuel. 
To complete the energetics for this target, we must include the initial drive energy required to compress the fuel before antiproton injection. The energy stored in the cold fuel results from the $p d V$ work done. If the fuel is maintained at its minimum Fermidegenerate electron pressure, the energy per unit volume is then just the product of the electron number density $n$ and the average energy per particle $0.6 \varepsilon_{\mathrm{F}}$, where $\varepsilon_{\mathrm{F}}$ is the Fermi energy $\approx 3.65 \times 10^{-15} n^{2 / 3} \mathrm{eV}$ with $n$ in $\mathrm{cm}^{-3}$. We then estimate the compression energy in the fuel in $\mathrm{J} / \mathrm{cm}^{3}$ as a function of uranium seed fraction $f_{u}$ as approximately:

$$
E_{\text {comp }}\left(f_{u}\right) \approx 3.5 \times 10^{-34} \alpha n_{H}^{5 / 3}\left(1+z_{U} \frac{f_{u}}{1-f_{u}}\right)^{5 / 3}
$$

where $n_{H}$ is the $\mathrm{D}+\mathrm{T}$ number density in $\mathrm{cm}^{-3}$ and $\alpha$ is the ratio of the fuel pressure to the ideal Fermi pressure and accounts for the fact that the fuel is not perfectly Fermidegenerate. The second term is an estimation of the degeneracy pressure contribution due to electrons from the uranium seed. The ionization state is approximated as $z_{U} \approx 1.03 T(\mathrm{eV})^{0.45}[2]$ and we take $\mathrm{T} \sim 0.6 \varepsilon_{\mathrm{F}}$. The total driver energy is then dependent on the efficiency of coupling of the driver energy to the capsule, $\eta_{d}$, and the efficiency of the ablation-driven compression, $\boldsymbol{\eta}_{\text {hydro }}$, as

$$
E_{\text {driver }}=\frac{E_{\text {comp }}}{\eta_{d} \eta_{\text {hydo }}}
$$

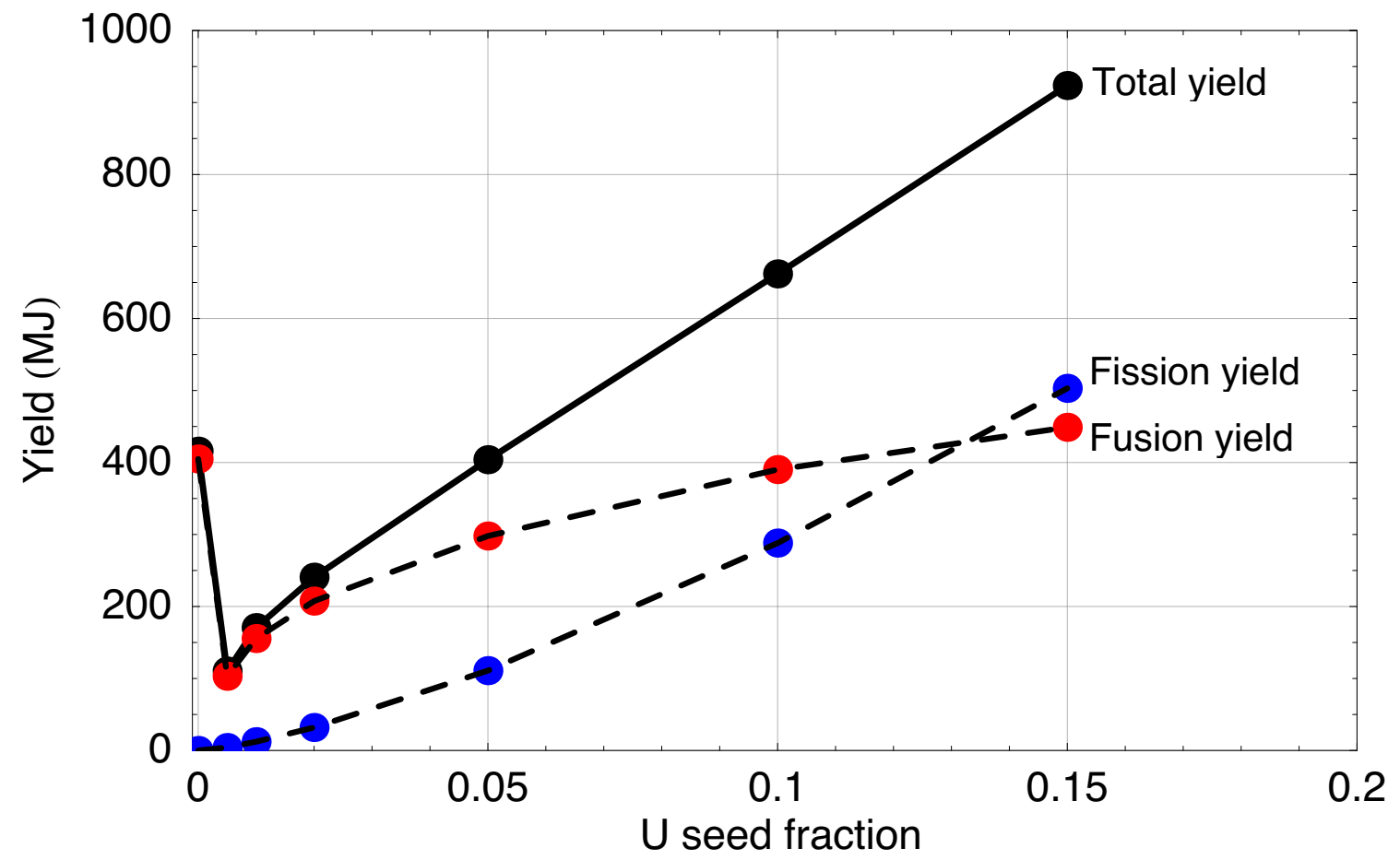

Fig 10. Partition between fusion and fast fission yields in volumetric ignition as the uranium seed fraction is increased. The fission yield exceeds the fusion yield for $f_{u}>13 \%$

Applying Eqn. 9 with $\alpha=2$, the fuel compression energy would increase from $\sim 90 \mathrm{~kJ}$ for pure DT through $\sim 380 \mathrm{~kJ}$ at a seed fraction of $f_{u}=0.10$. Given that advanced, reactor-scale (conventional) drivers might approach efficiencies of $\boldsymbol{\eta}_{d} \boldsymbol{\eta}_{\text {hydro }}, \sim 15-20 \%$, we 
would require compression driver energies over a megajoule for higher seeded targets before the antiproton volumetric heating source is applied. Accordingly, for space applications, the parallel use of antiproton-driven ablation drive should be considered; this is described in Section 5 below.

Overall burn energetics from Fig. 10 show that the fusion yield is saturating at $\sim 460 \mathrm{MJ}$ at higher seed fractions. Because there is a fixed $5 \mathrm{mg}$ DT fuel mass, this represents an ultimate fusion burn fraction of $\sim 0.27$. At $f_{u}=15 \%$, the uranium fuel mass is $84 \mathrm{mg}$, so that the specific uranium fission yield is $\sim 6000 \mathrm{MJ} / \mathrm{g}$ and around $7 \%$ of the ${ }^{238} \mathrm{U}$ is fissioning. Given that uranium is dominating the target mass at higher seed fractions, the specific fission yield per unit mass is effectively constant and the corresponding fission burn fraction will saturate around $\sim 10 \%$. Thus, from space propulsion viewpoint, constant exhaust velocities would also be obtained.

\subsection{Volumetric Ignition Design Point}

As an example design point, we take a capsule with $5 \%$ uranium seed. The mass of the fuel capsule is now dominated by the additional $25 \mathrm{mg}$ of ${ }^{238} \mathrm{U}$. Igniting this capsule at $10 \mathrm{keV}$ would result in a total yield of $\sim 405 \mathrm{MJ}$ with $27 \%$ coming from fission (Fig. 10). The total number of incident antiprotons required to drive this capsule is $\sim 6.5 \times 10^{17}$ resulting in an overall gain of $\sim 2$. The separate compression energy would be $\sim 200 \mathrm{~kJ}$ at $\alpha=2$ requiring a driver of $\sim 1.3 \mathrm{MJ}$ at $\eta_{d} \eta_{\text {hydro }}, \sim 15 \%$. Given the large number of antiprotons required to drive this class of target and the resulting low gains, we conclude below that $\overline{\mathrm{p}}$-driven fission-fusion volumetric ignition targets offer little promise compared to other potential options.

For space propulsion applications, the average exhaust velocity provided by the target after ignition and burn has occurred is

$$
<V_{\begin{array}{c}
\text { rocket } \\
\text { exhaust }
\end{array}}>\approx \sqrt{\frac{2 E_{\text {ch arged }} \varepsilon_{J E T}}{m_{\text {fuel }}}}
$$

where $E_{\text {charged }}$ is the energy from the fission+fusion target yield in charged particle form which is this is distributed over escaping prompt fusion burn products and thermalized target debris, and $\varepsilon_{\mathrm{JET}}$ is the jet efficiency for the spacecraft's engine and nozzle systems. The latter accounts for the projection of velocities onto the rearward thrust vector, as well as other efficiencies in forming the exhaust. From the LASNEX burn calculation, $215 \mathrm{MJ}$ of the $404 \mathrm{MJ}$ total yield appears as charged particles including all of the $27 \%$ fission yield; the balance is lost as escaping fast neutrons which do not contribute to directed thrust. The resulting average particulate velocity is $\sim 3.8 \times 10^{6} \mathrm{~m} / \mathrm{s}$ with an average particle energy of $\sim 1.06 \mathrm{MeV}$. Such particles must be directed into a rearward exhaust, which introduces the above jet-efficiency factor.

Because of the low target mass, the impulse per target is only $\Delta m v=F . \Delta t$ $\sim 113 \sqrt{ } \varepsilon_{\text {JET }}$ N.s which, at a rep-rate of, say, $30 \mathrm{~Hz}$ translates to a thrust of $\sim 3400 \sqrt{ } \varepsilon_{\text {JET }} N$. Such targets would be employed in the later stages of a mission where high exhaust velocities are required. For earlier stages, a propellant mass $m_{\text {prop }}$ could be added to the 
target to increase thrust while matching the exhaust velocity to the optimum required to leave the exhaust at rest in the mission center-of-mass. In such a case, the thrust and exhaust velocity would scale as $\sim \sqrt{ } m_{\text {prop }}$ and $\sim 1 / \sqrt{ } \mathrm{m}_{\text {prop }}$ respectively. For targets described below, such mass could be in the form of a thick hohlraum case plus up to $\sim 50 \mathrm{~g}$ of mass surrounding the target (i.e., expellant). For large payload missions, larger yield targets would be required [9]. Accordingly, gain scaling is discussed in Section 6 below.

Note, that greater than $1 \mathrm{GJ}$ yields will result for seed fractions in excess of 0.17 , with $>55 \%$ of the energy coming from fission. One advantage of running a volumetrically driven target in this fission-dominated mode at high $f_{u}$ is that approximately the same number of antiprotons in the range $\sim 9 \times 10^{17}$ are required to initiate such targets, effectively independent of the initial mass (Fig. 8) - at least up to seed fractions of a few tens of percent - with a corresponding increase in gains and yields. Of course, more compression energy is required because of the higher initial uranium density (i.e., higher mass in the same compressed radius). For example at $f_{u}=0.17 \%(\sim 1 \mathrm{GJ}$ total yield) the compression energy is $\sim 400 \mathrm{~kJ}$, which requires an additional $2.7 \mathrm{MJ}$ driver at $\eta_{d} \eta_{\text {hydro }}, \sim 15 \%$.

\section{ANTIPROTON-DRIVEN HOTSPOT IGNITION}

\subsection{Hotspot Ignition Physics}

Here we examine ICF hotspot ignition with direct $\overline{\mathrm{p}}$ ablative drive. The principle was introduced in Section 3.3 above and shown in Fig. 4. As with volumetric ignition, a heavy metal seed is required to enhance antiproton energy deposition. In this case the ${ }^{238} \mathrm{U}$ seed is placed only in the ablator and not in the fuel itself. This results in clean DT fuel and, because only a small fraction of the fuel is initially heated to ignition temperatures, less incident antiprotons will be required per target resulting in higher gains. However, unlike the volumetric target, there will be no effective contribution to the target yield from fusion-induced fission because the uranium seed in the ablator will have blown out to low densities before the DT fuel ignites.

The target design consists of a central region of low density DT gas surrounded by a thin spherical shell of solid DT fuel and an outer ablator (Fig. 4). We have selected

${ }^{238} \mathrm{U}$-seeded DT as the ablation material for analysis but combinations of the heavy metal with other light matrix materials - e.g., CH plastic, beryllium, etc - are possible candidates. A fraction of the antiproton annihilation energy is deposited in the ablator and, as shown below, the heated plasma expands outwards with velocities of $\lesssim 10^{7} \mathrm{~cm} / \mathrm{s}$; these are somewhat lower exhaust velocities than encountered for conventional laser direct drive. By momentum conservation, the remaining portion of the capsule is driven inwards, compressing and heating the DT fuel via shocks and $P d V$ work, and, at peak antiproton drive power - that is, peak injection rate - an approximately uniform acceleration should pertain.

After peak implosion velocity, the shell stagnates on the gas at the center, converting kinetic energy to pressure. The now subsonic capsule relaxes to an approximate pressure equilibrium with a high temperature, low density hotspot comprising a few percent of the mass, surrounded by the bulk of the cold fuel at high 
density. If the hotspot can be created with a temperature of $T_{i g n} \gtrsim 10 \mathrm{keV}$ over a region of local areal density of $(\rho R)_{i g n} \sim 0.3 \mathrm{~g} / \mathrm{cm}^{2}$, then energy deposition per unit volume from energetic $3.5 \mathrm{MeV}$ alpha particles from the DT fusion reaction will exceed energy loss from Bremsstrahlung and electron heat conduction. Under such conditions, a thermonuclear burn wave can propagate radially outwards into the cold dense fuel with the overall burnup determined by the total $\rho R$ of the assembled fuel [2]. If the ablator has been completely ablated at the time of peak implosion velocity and the fuel is kept nearly isentropic, Herrmann et al [11] have shown that the implosion dynamics can be characterized by four parameters: the fuel mass, the ablation pressure, the in-flight adiabat (i.e., the ratio of the in-flight fuel pressure to the irreducible Fermi degenerate pressure) and the peak implosion velocity. In particular, several studies have highlighted the critical dependence of ignition threshold on the achievable peak implosion velocity $[2,11,64-66]$. The two major parameters of interest here for antiproton-drive hotspot ignition, i.e., ignitability and yield, are then a function of these four parameters and, therefore, on the number of injected antiprotons.

In general, the energy that must be delivered to a spherical capsule to create ignition conditions scales as

$$
E_{i g n} \sim \frac{\alpha^{x_{1}}}{v^{x_{2}} \eta_{\text {hydro }}}
$$

where $v$ is the peak implosion velocity, $\alpha$ is the fuel adiabat parameter and $\eta_{\text {hydro }}$, is the hydrodynamic efficiency of coupling the absorbed drive energy into stored compression energy $[2,11,64-66]$. Based on the two region, isobaric target model of Meyer-ter-Vehn [67], an early study [64] suggested exponents of $x_{1} \sim 3$ and $x_{2} \sim 10$. Numerical calculations by Levedahl and Lindl [65] that accommodated 1-D transport refined this to $x_{1} \sim 3 / 2$ and $x_{2} \sim 5$. Recent work by Herrmann et al. [11] et al for a suite of LASNEX runs showed that $x_{1} \sim 2.66$ and $x_{2} \sim 7.21$ providing $\alpha$ is taken to be the adiabat in the stagnated fuel (typically greater than the in-flight adiabat $\alpha_{i f}$ due to the interaction of reflected shocks). By explicitly including the drive pressure $P$, they arrived at a general ignition scaling law in terms of $\alpha_{i f}$ as

$$
E_{i g n} \sim \frac{\alpha_{i f}^{1.88}}{v^{5.89} P^{0.77} \eta_{\text {hydro }}}
$$

Note the high inverse dependence of ignition energy on achievable peak velocity. It is now believed that to maintain targets on acceptably low adiabats, peak implosion velocities obtainable with conventional drivers will be limited to about $3-4 \times 10^{7} \mathrm{~cm} / \mathrm{s}$ because of hydrodynamic instabilities and maximum attainable drive pressures [2].

\subsection{Analysis and Results}

In this study, we will not resort to a full 1D radiation-hydrodynamics-burn calculation, not least because we have no formal models for antiproton ablation drive in 
our present codes. Instead, because of the primacy of implosion velocity in determining ignitability (Eqn. 12), we model the dependence of this velocity on injected antiproton flux and thereby estimate the number of injected antiprotons required to drive a typical hotspot target. Provided the required drive pressure and peak velocity can be obtained, then, to zeroth order, we might expect the burn dynamics to proceed similar to that of a conventionally-driven target.

We take a $5 \mathrm{mg}$ DT fuel mass with inner radius of $0.178 \mathrm{~cm}$ and initial shell thickness of $0.040 \mathrm{~cm}$ at an uncompressed cryogenic solid density of $0.25 \mathrm{~g} / \mathrm{cm}^{3}$. These dimensions were scaled from a high gain reactor target we are presently studying for conventional direct drive [6]. The fuel is surrounded by a ${ }^{238} \mathrm{U}$-seeded DT ablator of shell thickness $0.032 \mathrm{~cm}$ with DT mass of $5.4 \mathrm{mg}$. The kinetic energy of the incident antiprotons is adjusted so that they have a slowing down range in the ablator equal to the average fission product range $<R_{f p}>$ of the fragmenting uranium seed nuclei. The thickness of the annihilation energy deposition region and therefore, the approximate thickness of the ablation front, is then $\sim 2<R_{f p}>$. By analogy with Eqn. 6 above, the energy deposited in the ablation front per antiproton as a function of the uranium seed fraction $f_{u}$ is then approximately

$$
E_{a}\left(f_{u}\right) \approx p_{u}\left(f_{u}\right) p_{f}<E_{f}>+\left(1-p_{u}\left(f_{u}\right)\right) \frac{\rho_{a}<R_{f p}>}{(\rho R)_{\pi}}<E_{\pi}>
$$

where $\rho_{a}$ is the average density across the ablation front.

We estimate the range $\left\langle R_{f p}>\right.$ of antiproton-induced fission fragments in the seeded ablator using a empirical fit to fission product range data $[68,69]$ as

$$
<R_{f p}>\approx\left(0.0968+0.0497 \frac{M}{z^{1 / 2}}\right) \frac{M_{f}^{1 / 2}<E_{f}>^{1 / 2}}{z_{f}^{3 / 2} \rho_{a}}
$$

where $M$ and $z$ are the mass number and atomic number of the stopping medium, and $M_{f}$ and $z_{f}$ are the mass number and atomic number (i.e., bare charge) of the fission fragment. This is an average over all light and heavy fragments characterized by an average energy $\left.<E_{f}\right\rangle$. The range $\left\langle R_{f p}>\right.$ is in units of $\mathrm{cm}$ for $<E_{f}>$ in Mev and $\rho_{a}$ in $\mathrm{mg} / \mathrm{cm}^{3}$.

The charge state of the fission fragment as it slows is accommodated by the empirical fit coefficients of Eqn. 14 but is taken to scale with velocity $v_{f}$ as $q\left(v_{f}\right) \sim 20\left(v_{f} / v_{f o}\right)^{1.1}$ where $\mathrm{v}_{f o}$ is the initial velocity corresponding to an energy $\left\langle E_{f}>[68]\right.$.

As an example, in Fig. 11 we plot the ranges of antiproton-induced ${ }^{238} \mathrm{U}$ fission fragments and DT annihilation pions in the ablation front as a function of the ablator seed fraction. The methodology for determining pion ranges was described in Section 4 above. The ranges are shown for densities of one and twenty times solid density; such densities span the time-dependent range we typically see at the ablation front during 1-D compressions of conventional high yield reactor targets [6,70]. From Fig. 11, assuming that fission product deposition is dominating the net energy deposition (a good assumption for $f_{u}>1 \%$ - see Fig. 7 above) the thickness of the antiproton-driven ablation front is seen to be in the vicinity of ten microns, comparable to that of a conventional, 
radiation-ablated indirect drive target [70]. The antiproton injection kinetic energy required to penetrate to the center of the ablation front, i.e., a stopping range of $2<R_{f p}>/ 2$ can be calculated by Eqn. 8 and evaluates to $\sim 1.4 \mathrm{MeV}$, more than two orders of magnitude smaller than the typical net deposited annihilation energy. The injection energy is only a weak function of increasing seed fraction as both the antiproton range and fission product range scale similarly with $f_{u}$.

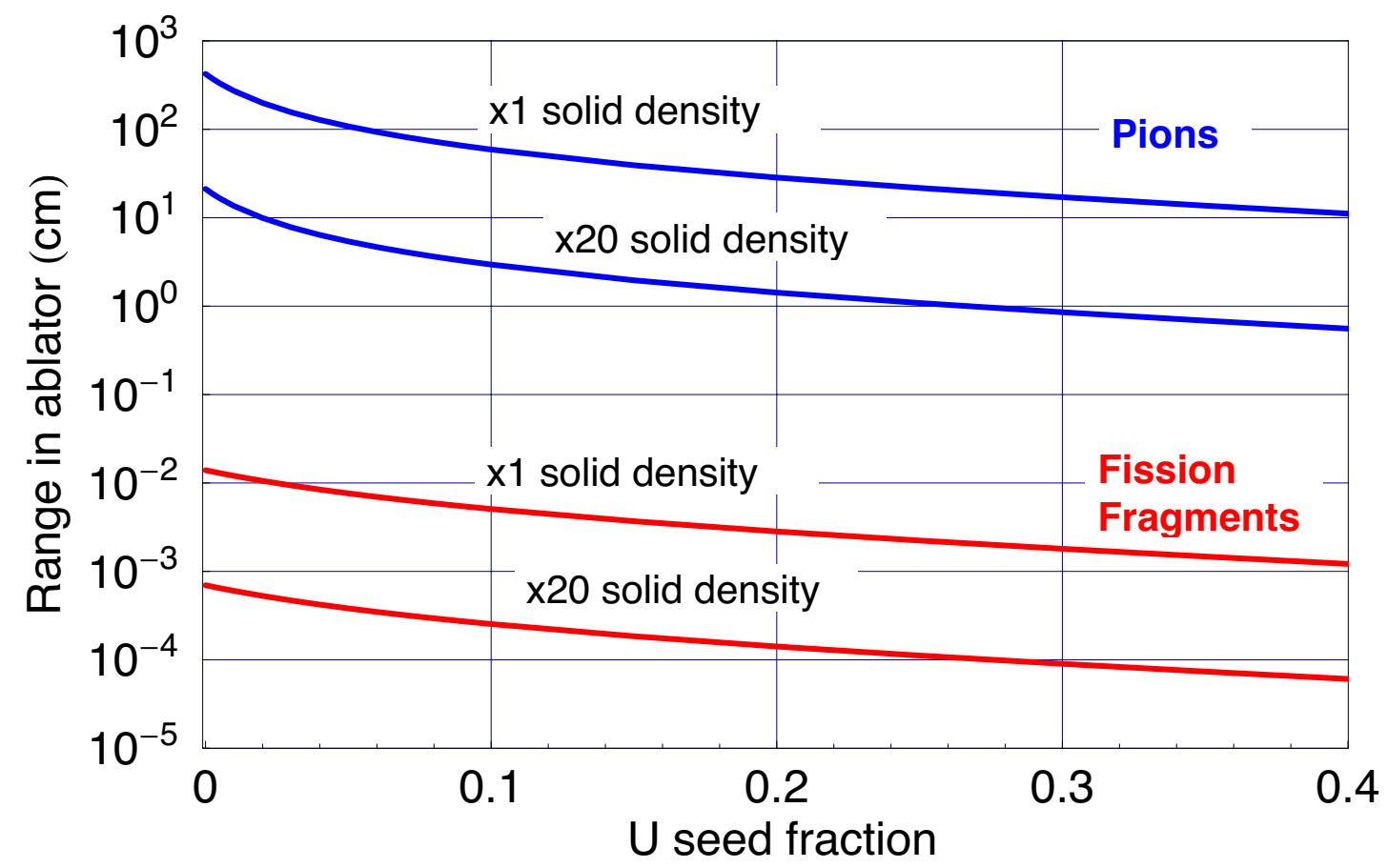

Fig. 11 Range of annihilation pions and fission fragments in the DT/U ablator versus ablator uranium seed fraction for $x 1$ and $x 20$ solid density.

With the formalism for antiproton energy deposition in the ablation front, we can now estimate peak shell implosion velocities as a function of the number of incident antiprotons by integrating the rocket equation for momentum conservation:

$$
v=\int_{m_{o}}^{m_{f}} v_{e x h}(t) \frac{d m}{m(t)}
$$

where $m_{o}$ is the initial total mass of the DT fuel and ablator and $m_{f}$ is the final mass of the fuel. We set the exhaust velocity as the ratio of ablation pressure $P$ to mass ablation rate per unit area $m_{A}$, and take this pressure as the ratio of the drive intensity to the blowoff velocity $v_{e x h}$ such that

$$
v_{e x h}=\frac{P}{m q_{A}} \approx \sqrt{\frac{I}{m q_{A}}}=\sqrt{\frac{\phi E_{a}}{m q_{A}}}
$$

where the intensity $I$ is simply the product of the antiproton flux per unit area per second $\phi$ and the energy deposited in the ablator per antiproton $E_{a}$ from Eqn. 13. The integration of Eqn. 15 requires a time dependent knowledge of the incident flux and the ablator 
density $\rho_{a}$. As we are only seeking typical integral performance parameters and are not performing a self-consistent $1 \mathrm{D}$ compression, we do not attempt to formally evaluate a temporal shape for the antiproton pulse. Instead, we assume a generic pulse shape and time-dependent average density across the ablation front as:

$$
\begin{aligned}
& \phi(t)= \begin{cases}0.08 \not \partial & 0<t \leq t_{1} \\
\phi\left(0.92 \frac{t-t_{1}}{t_{2}-t_{1}}+0.08\right) & t_{1}<t \leq t_{2} \\
\phi & t_{2}<t \leq t_{3}\end{cases} \\
& \rho_{a}(t)=\rho_{a 0}(1.06)^{t} \quad 0 \leq t \leq 40 n s
\end{aligned}
$$

these being scaled from a representative 1-D simulation of a conventional indirect-drive capsule with comparable dimensions and total yield and approximately comparable exhaust velocities and ablation front widths [70]. Here $t_{1}=24 \mathrm{~ns}, t_{2}=32 \mathrm{~ns}, t_{3}=40 \mathrm{~ns}$ and $\rho_{a 0}$ is the uncompressed density of the ablator at $\mathrm{t}=0$. The time integral of Eqn. 17 therefore yields the total number of antiprotons required per unit area to drive the capsule to a peak implosion velocity $v$ evaluated by Eqns. 15 and 16 .

In Fig 12 we plot contours of peak implosion velocities attainable for a given total number of incident antiprotons as a function of the uranium seed fraction in the ablator. Note the very large number of antiprotons required at low seed fraction because of the minimal energy deposition of annihilation pions in the ablator. We might also expect significant relative preheating of the fuel by these pions in this regime that may preclude high fuel compression on a low adiabat. Given the achievable velocity is a balance between adequate energy deposition in the ablator and decreasing exhaust velocity at higher ablator masses, we see a broad maximum in the peak inward shell velocity for a fixed number of injected antiprotons around a seed fraction of $f_{u} \sim 0.2$.

The corresponding average ablator exhaust velocities can be mapped onto the space of Fig. 12 and these are shown in Fig.13. Here, for a given antiproton number, maximum exhaust velocities are attainable for seed fraction of $f_{u} \sim 0.03$ where the rocket efficiency is a maximum (see below). The broad maximum in peak implosion velocities at the higher 0.2 seed fraction seen in Fig 12 above then results from the logarithmic dependence on ablated mass from the rocket equation, i.e., $v \sim<v_{e x h}>\ln \left(m_{0} / m_{f}\right)$

\subsection{Hotspot Ignition Design Point}

We can obtain an approximate design point by selecting a desired peak shell velocity of, say, $3 \times 10^{7} \mathrm{~cm} / \mathrm{s}$. The kinetic energy at peak velocity for the $5 \mathrm{mg}$ fuel shell is then $\sim 2.25 \times 10^{5} \mathrm{~J}$. If we assume that this stagnates into a hotspot at the ignition temperature of $T_{i g n} \sim 10 \mathrm{keV}$ containing approximately $2 \%$ of the total fuel mass, typical for an isobaric hotspot target [67], then the energy in the hotspot is $\sim 1.15 \times 10^{5} \mathrm{~J}$ leaving $\sim 1.1$ $\mathrm{x} 10^{5} \mathrm{~J}$ compressional stored energy in the surrounding cold fuel. At a stagnation adiabat of $\alpha_{\text {stag }} \sim 3$, the target would have an areal density of $\rho R \sim 3$ and, by Eqn. 1, a fuel burnup of around one third. For 5mg of DT, this represents a fusion yield of $\sim 560 \mathrm{MJ}$. 


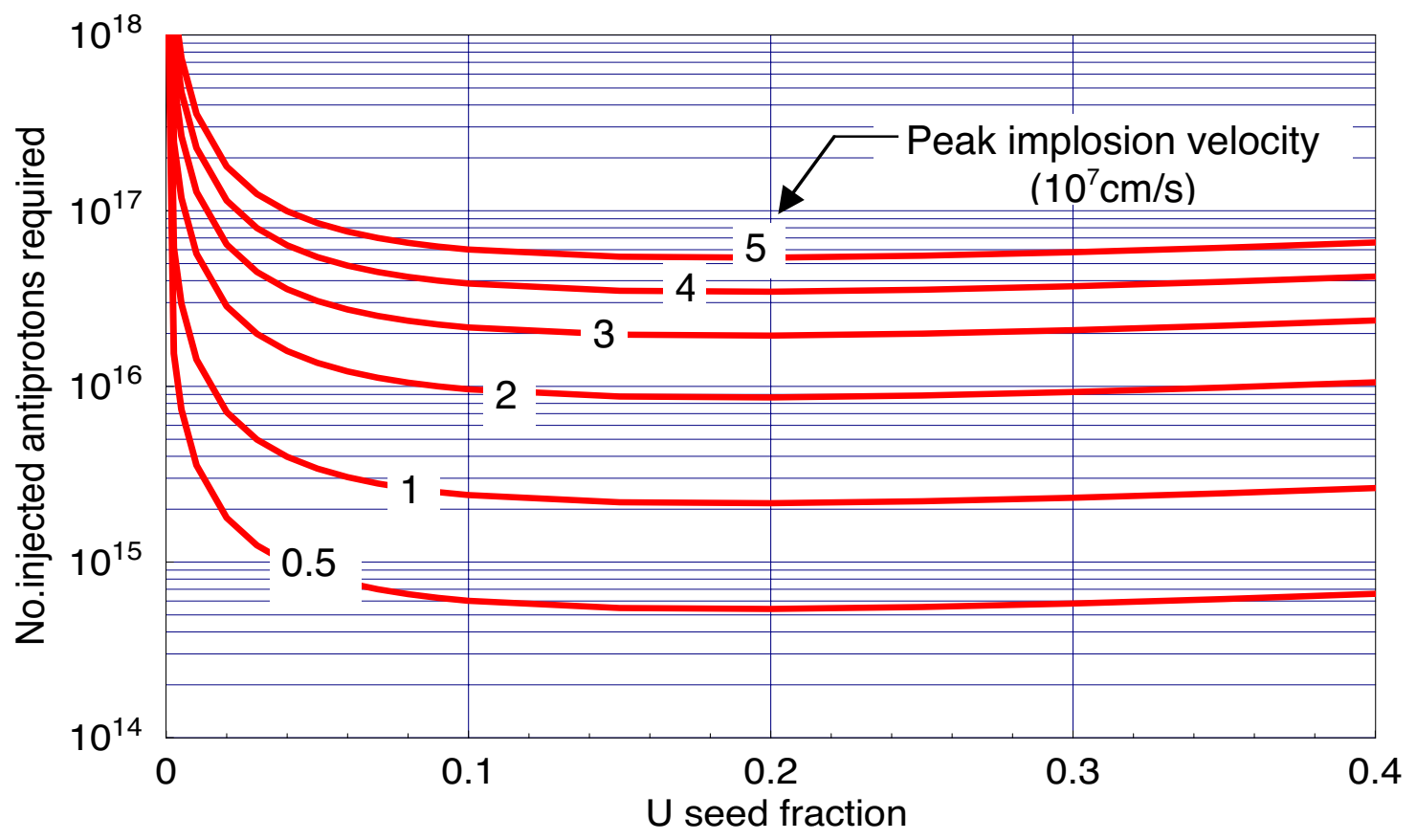

Fig. 12 Contours of peak shell implosion velocity as a function of number of injected antiprotons and ablator uranium seed fraction. A broad minimum in the required number of antiprotons for a given peak velocity is seen around $f_{u} \sim 20 \%$

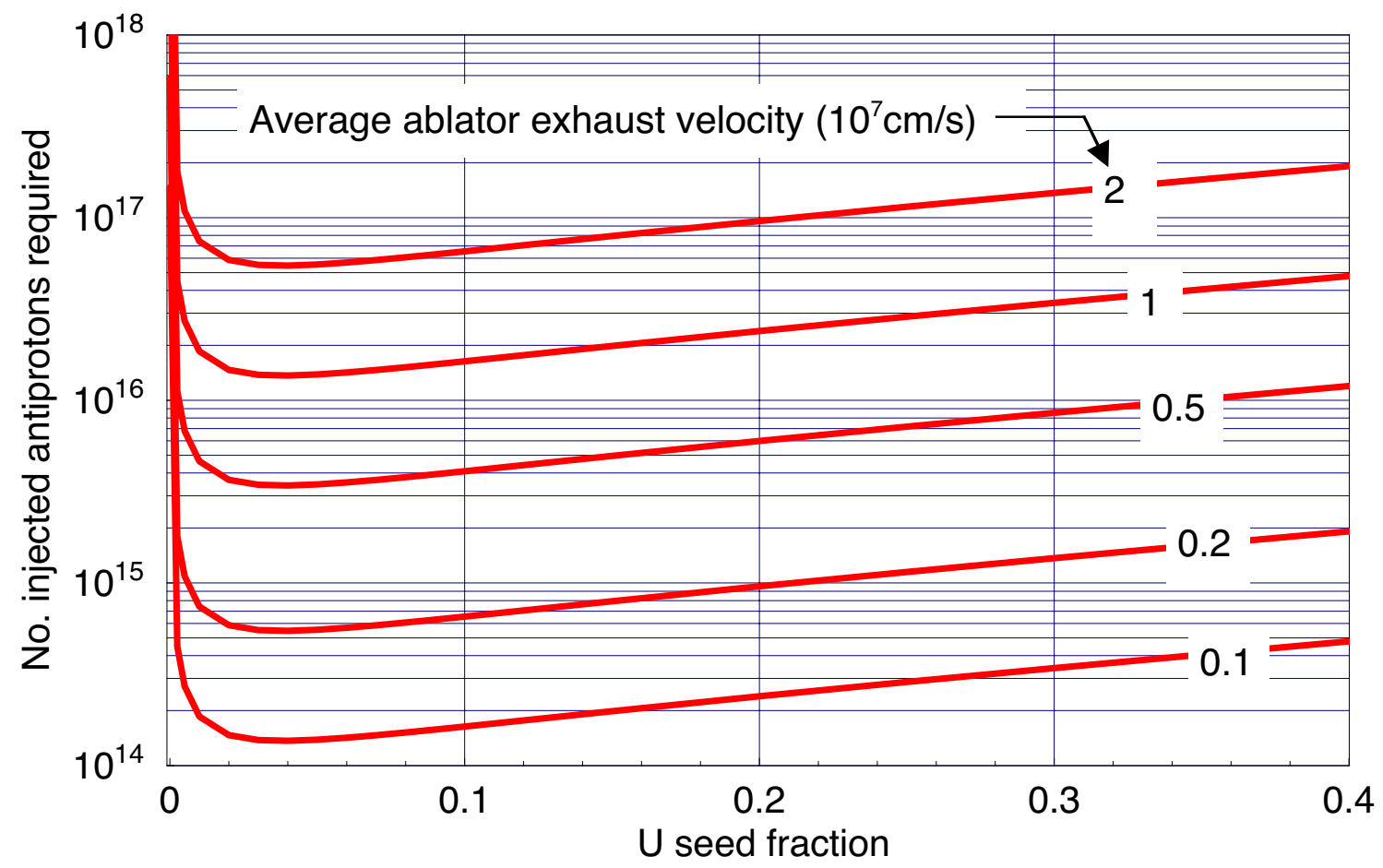

Fig. 13 Contours of average ablator exhaust velocity as a function of number of injected antiprotons and ablator uranium seed fraction. Exhaust velocities maximize for a given injected antiproton number around $f_{u} \sim 3 \%$ where the rocket efficiency is a maximum 
In Fig. 14, we plot a gain curve for the fixed fuel mass of $5 \mathrm{mg}$ as a function of ablator seed fraction by using data corresponding to the $v=3 \times 10^{7} \mathrm{~cm} / \mathrm{s}$ contour in Fig 12, and where we define gain as the fusion yield from above $(\sim 560 \mathrm{MJ}$.$) divided by the total$ rest mass energy available in the injected antiprotons $(1.88 \mathrm{GeV}$ per antiproton). We also plot the ideal hydrodynamic (rocket) efficiency in Fig. 14 where

$$
\left.\eta_{\text {hydro }}\left(f_{u}\right) \approx \frac{\left(m_{f} / m_{o}\left(f_{u}\right)\right) \ln \left[m_{f} / m_{o}\left(f_{u}\right)\right]^{2}}{1-m_{f} / m_{o}\left(f_{u}\right)}\right|_{v=3 \times 10^{7} \mathrm{~cm} / \mathrm{s}}
$$

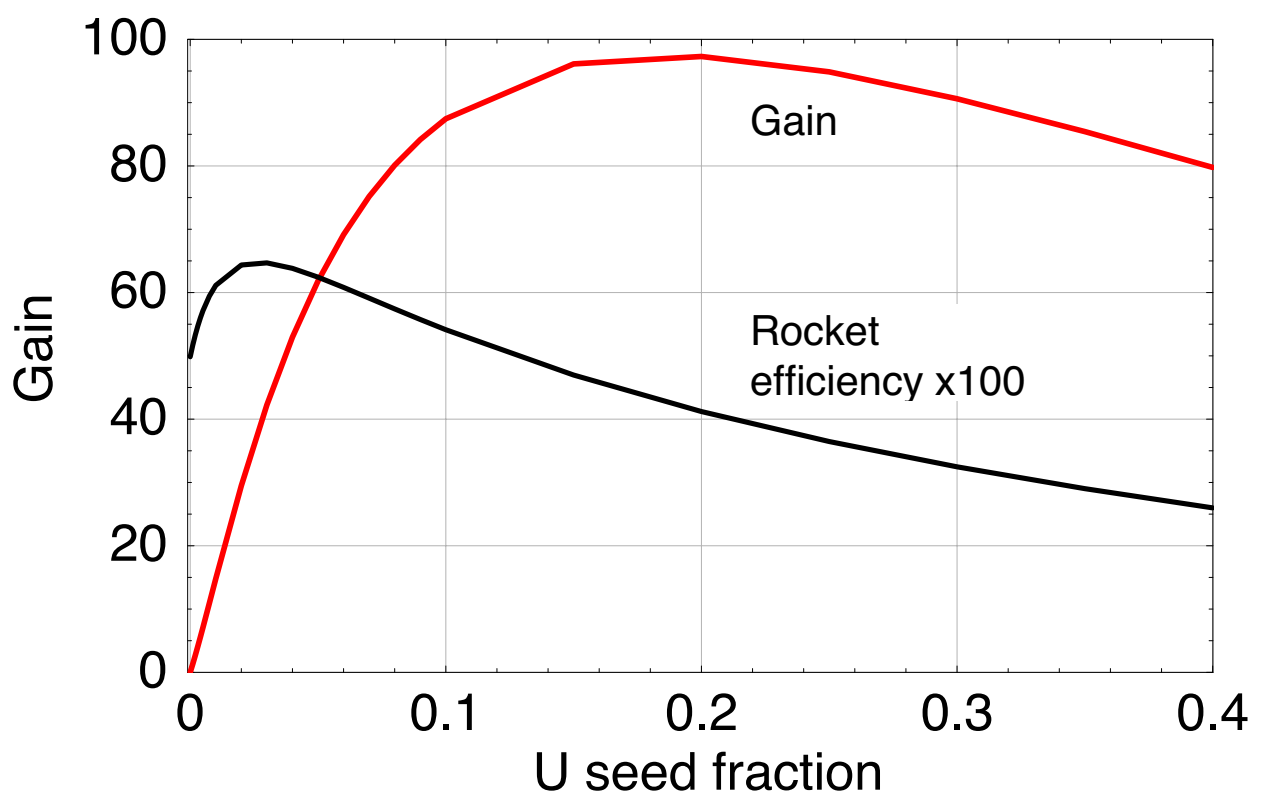

Fig 14. Gain curve and ideal hydrodynamic (rocket) efficiency for antiproton-driven hotspot ignition at $3 \times 10^{7} \mathrm{~cm} / \mathrm{s}$ peak implosion velocity as a function of ablator uranium seed fraction. Peak gains approach 100 at $f_{u} \sim 20 \%$. (Peak gains for fast ignited targets are $\sim 600$ at the same ablator seed fraction - see below). The rocket efficiency is a maximum at $f_{u} \sim 3 \%$ where maximum exhaust velocities are obtained

Antiproton drive should be closer to this ideal efficiency than conventional drivers because, unlike laser or radiation drive, minimal kinetic energy is expended in transiting the ablation exhaust relative to the annihilation energy deposited at the ablation front. In particular, the antiproton kinetic energy lost in penetrating to the ablation front is only $\sim 1.4 \mathrm{MeV}$, whereas the energy deposited there is some $10-20 \%$ of the total $1.88 \mathrm{GeV}$ annihilation energy, depending on seed fraction (see Fig. 7). Radiation energy loss at higher uranium seedings will, however, reduce actual efficiencies from these ideal values.

The peak gain occurs at a seed fraction of $f_{u} \sim 0.2$ where the minimum number of antiprotons is required for a given peak implosion velocity (see Fig.12). By contrast, the maximum rocket efficiency occurs at a seed fraction of only $\sim 0.03$ where exhaust 
velocities maximized (see Fig. 13), a consequence of the fact that hydrodynamic efficiency is defined in terms of antiproton annihilation energy deposited, while gain is defined in terms of antiproton annihilation energy injected. As shown, peak gains approaching 100 may be obtainable for this hotspot target- an order of magnitude higher than those for volumetric ignition above - but still requiring the injection of some $2 \times 10^{16}$ antiprotons per target (Fig. 12). Scaling of the targets to higher yields and gains will be discussed below.

\subsection{Target Stability}

For a given antiproton-driven ablation pressure $P$, a larger radius, thinner shell enclosing more volume could be driven to a higher final velocity than a thicker shell of the same mass. However, there is a limit on the aspect ratio of the shell thickness to radius determined by the Rayleigh-Taylor fluid instability [2,71]. If the in-flight aspect ratio (IFAR), is defined as the ratio of the shell thickness $\Delta R_{o}$ to its radius $R_{0}$ as it begins to accelerate (this is less than the initial thickness because the shell is subsequently compressed) then, using the scalings in Ref. 2, this can be related to the implosion parameters above as

$$
I F A R \equiv \frac{\Delta R_{o}}{R_{o}} \approx C v^{2} \alpha_{i f}^{-3 / 5} P^{-2 / 5} \approx C v^{2} \alpha_{i f}^{-3 / 5}\left(\frac{\phi E_{a}}{v_{e x h}}\right)^{-2 / 5}
$$

where $\mathrm{C}$ is a constant. Note the quadratic dependence on implosion velocity. Stability typically dictates an upper limit of $\sim 35-45$ on IFAR otherwise shell breakup will occur at late time and hotspot formation will be prevented.

We will not consider stability formally in this paper even though it is an important topic for overall target performance. In general, we note that the antiproton-driven implosions are high ablation rate, low exhaust velocity implosions. They are thus more akin to conventional radiation-driven, indirect drive implosions [70,72] than conventional laser direct-drive implosions [6,71] and have comparable ablation front thickness. Therefore, stability may be acceptable providing low mode asymmetries due to nonuniform antiproton injection can be minimized. If the latter proves problematic, then the semi-indirect-drive target outlined in Section 3.3 might be a better design candidate.

\section{ANTIPROTON-DRIVEN FAST IGNITION}

\subsection{Fast Ignition Rationale}

In the hotspot target above, the energy contained in the hotspot at ignition scales as the hotspot mass and temperature as $\sim R_{H S}^{3} \rho_{H S} T_{i g n}=(\rho R)_{i g n}^{3} T_{i g n} / \rho_{H S}^{2}$, i.e., inversely with the square of the attainable density for given ignition condition of $(\rho R)_{i g n}$ and $T_{i g n}$. By contrast, the compression energy in the fuel scales as $\sim \alpha \rho^{2 / 3}$ (Section 5). Thus, the sum of the ignition and compression energy could be reduced if the capsule is assembled to an approximately uniform density that is higher that the hotspot density but lower than 
the cold fuel density, followed by subsequent ignition of an outer portion of the fuel by a separate fast energy source.

Antiprotons could be used in fast ignition as the ablative drive system to compress the fuel in a manner similar to hotspot ignition above but with relaxed timing constraints. In principle, they could also be used as the fast energy source for the ignition spot. As shown below, the latter is faced with stringent requirements in terms of injection times and aiming precision, so antiprotons may have more utility to just drive the much relaxed, slow compression phase of fast ignition. In such case, a separate conventional fast energy source such as a short pulse laser would be needed to spark the ignition.

\subsection{Fast Ignition Hotspot Creation}

A propagating burn under the isochoric (constant density) conditions of fast ignition requires ignition conditions of $\mathrm{T}_{\mathrm{ign}} \sim 10 \mathrm{keV}$ over a local areal density of $(\rho R)_{i g n} \sim 0.5 \mathrm{~g} / \mathrm{cm}^{2}$ (Section 3.4). Once the density of the ignitor region is specified, this represents a specific deposited energy. Accordingly, the required number of antiprotons delivered to the ignitor spot to create these conditions is approximately:

$$
\left.N_{\bar{p}}\left(f_{u}\right)\right|_{\text {fast }} \approx 3 \times 10^{21} \frac{(\rho R)_{i g n}^{3} T_{i g n}}{\rho_{H S}^{2}\left(f_{u}\right) E_{n e t}\left(f_{u}\right)}
$$

where $E_{n e t}(\mathrm{Mev})$ is the net energy deposition in the hotspot per antiproton and where, for fast ignition, the hotspot density is of the order of the bulk fuel stagnation density $\rho_{H S} \sim \rho_{\text {stag }}\left(\mathrm{g} / \mathrm{cm}^{3}\right)$.

Heavy metal seeding of the ignitor core (but not the bulk fuel) is presumably required otherwise the local deposition of annihilation energy is minimal. At a seed fraction of $f_{u}=0.01$, the net deposition fraction per antiproton is $\sim 3.6 \%$, i.e. $70 \mathrm{MeV}$ (Fig 7). Thus, if the overall DT fuel is initially compressed to say, $100 \mathrm{~g} / \mathrm{cm}^{3}$ by the separate slow compression system, the ignitor core with its $1 \%$ uranium seed will be at $\rho_{H S} \sim 200 \mathrm{~g} / \mathrm{cm}^{3}$, and by Eqn. 21 will require a minimum $\sim 1.4 \times 10^{15}$ delivered antiprotons for a hotspot spark energy of $\sim 15 \mathrm{~kJ}$. A caveat, however, is that the minimum $\rho R_{i g n} T_{i g n}$ conditions for fast ignition burn propagation may be higher with heavy metal seeding present due to increased Bremsstrahlung losses from the hotspot. Secondly, ionization of the $1 \%$ uranium seed at the ignition temperature of $10 \mathrm{keV}$ will contribute additional electrons to the hotspot, thus increasing the heat capacity over that implied in Eqn. 21. (e.g., with the estimation of the ionization state of uranium $z_{U}$ used in Eqn 9 above, the $z_{\text {eff }}$ of the hotspot will be $\sim 1.6$ at $f_{U}=1 \%$ and the delivered antiprotons requirement would increase to $\sim 2.2 \times 10^{15}$ ).

\subsection{Slow Compression Drive}

We now examine how antiprotons could be applied to drive the slow compression phase of fast ignition. The fast ignition source could then be either a fast antiproton burst as described above, or a conventional fast energy source such as a short pulse laser. As with hotspot ignition above, we take the peak attainable shell velocity as the datum parameter of interest. Here, however, the shell kinetic energy is only required to convert 
to a rather modest stagnation density under isochoric conditions, rather than to create the ignition hotspot energy and high density compressed fuel.

In their study of radiation-driven capsules for fast ignition, Slutz and Herrmann [53] provide a scaling of stagnation density in $\mathrm{g} / \mathrm{cm}^{3}$ as

$$
\rho_{\text {stag }} \approx 6 \times 10^{-20}\{v / \sqrt{\alpha}\}^{3}
$$

where $\mathrm{v}$ is the peak implosion velocity in $\mathrm{cm} / \mathrm{s}$. This is obtained by taking the peak kinetic energy of the imploding shell to be adiabatically converted to internal energy. It assumes a uniform final density whereas hollow profiles may be hard to avoid in practice [73]

Peak shell velocities for antiproton ablative drive to achieve this stagnation density can then be obtained by the methodology of Section 5 as a function of the number of incident protons. Thus, in general, the total optimum number of antiprotons to ignite and burn a fast ignition capsule is a minimization over those required for both the slow compression drive and the fast ignition spark as:

$$
N_{\bar{p}}\left(\rho_{\text {stag }}, f_{u, a}, f_{u, s}\right)=\left.N_{\bar{p}}\left(\rho_{\text {stag }}, f_{u, a}\right)\right|_{\text {comp }}+\left.N_{\bar{p}}\left(f_{u, s}\right)\right|_{\text {fast }}
$$

where $\rho_{\text {stag }}$ is the fuel stagnation density, and $f_{u, a}, f_{u, s}$ are the seed fractions in the ablator and fast ignition core, respectively.

\subsection{Fast Ignition Design Point}

As an example of applying Eqn. 23, we take a design similar to the hotspot capsule above with 5mg of DT fuel - unseeded, except for the designated hotspot region if antiproton fast ignition is also to be employed - and an outer ${ }^{238} \mathrm{U}$-seeded DT ablator (Fig. 5). We appreciate that it may be difficult in practice to seed the hotspot region and not seed the main fuel, both as an issue of itself and as something that might affect the symmetry of the compression. We take a stagnation fuel density of $\rho_{\text {stag }}=150 \mathrm{~g} / \mathrm{cm}^{3}$ which, by Eqn (22), necessitates a peak implosion velocity of $\sim 1.6 \times 10^{7} \mathrm{~cm} / \mathrm{s}$ at, say, $\alpha=1.5$. From the methodology of Section 5 above, this velocity can be obtained with a minimum number of $\sim 2.5 \times 10^{15}$ injected antiprotons at an optimum ablator seed fraction of $f_{u, a} \sim$ 0.2 (Fig. 12). From Eqn. 21, the fast ignition of the hotspot - assumed to be separately seeded at, say, $f_{u, s}=0.01$ for a total density of $\rho_{H S} \sim 300 \mathrm{~g} / \mathrm{cm}^{3}$ - requires $\sim 0.62 \times 10^{15}$ fast injected antiprotons.

The total of $\sim 3.1 \times 10^{15}$ fast and slow injected antiprotons for this fast ignition target is a factor of six lower in number and rest mass energy than that required for the hotspot ignition capsule above - or, a factor of eight if a separate, conventional energy source is employed to supply the fast ignition spark - and is demonstrative of the lower velocities required to assemble the fuel. Given that this target has around the same total areal density of $\rho R \sim 3 \mathrm{~g} / \mathrm{cm}^{2}$ as the hotspot capsule (because of the density gradient of the latter) and, therefore, around the same fusion yield of $\sim 560 \mathrm{MJ}$, the fast ignition gains will be higher by the same ratio. In this case, the ratio of fusion yield to the total energy 
annihilation available in the delivered antiprotons would be $\sim 600$, up from the gain of $\sim 100$ for hotspot ignition.

The isotropic particulate velocity of this target for space propulsion applications is $\sim 8.0 \times 10^{6} \mathrm{~m} / \mathrm{s}$ with an average particle energy of $\sim 0.84 \mathrm{MeV}$. The corresponding target impulse is $\sim 40 \sqrt{\varepsilon_{\mathrm{JET}}}$ N.s which, at a rep-rate of, say, $30 \mathrm{~Hz}$ translates to a thrust of $\sim 1200 \sqrt{ } \varepsilon_{\mathrm{JET}} \mathrm{N}$. Addition of inert propellant mass $m_{\text {prop }}$ in the form of a thick hohlraum case (and perhaps up to $50 \mathrm{~g}$ of additional expellant) would scale the thrust and exhaust velocity as $\sqrt{ } m_{\text {prop }}$ and $1 / \sqrt{ } \mathrm{m}_{\text {prop }}$ respectively. The total target mass could be varied about some mean value thus offering a variable specific impulse rocket with optimum exhaust velocity.

High payload space missions will probably require higher yield targets (see, for example, Ref 9). For conventional hotspot targets, as the driver energy $E_{\text {driver }}$ increases, the minimum velocity required to ignite the capsule decreases. If the minimum velocity at any driver energy is exceeded, the capsule will still ignite but the gain - i.e., the ratio of the fusion yield to $E_{\text {driver }}$ - will drop. The optimum strategy implies operating near the minimum implosion velocity consistent with desired yield or driver size. Accordingly, optimum gain curves follow a typical scaling of $\sim E_{\text {driver }}^{\gamma}$ where $\gamma \approx 0.6-0.8[1,2]$. Our hotspot target in Section 5 and the slow compression phase of the fast ignition target here implode in a manner analogous to that of conventional high yield ICF capsules.

Furthermore, like laser or radiation drive, the physics of antiproton deposition for ablative compression is governed by micron-size scalelength processes at the ablation front, effectively independent of the total yield. Thus, to a zeroth order, we might expect that gains will scale in a manner similar to those for conventional targets, offering an approximate yield scaling with number of injected antiprotons $N_{\bar{p}}$ as

$$
Y\left(N_{\bar{p}}\right) \approx 560\left(\frac{N_{\bar{p}}}{N_{\bar{p} 0}}\right)^{1+\gamma}
$$

where $\mathrm{Y}$ is the fusion yield in $\mathrm{MJ}, \gamma \approx 2 / 3$ and $N_{\bar{p} 0} \approx 3.1 \times 10^{15}$ or $\approx 1.9 \times 10^{16}$ for $\overline{\mathrm{p}}$-driven fast ignition or hotspot ignition targets, respectively

In the VISTA ICF space propulsion study of Ref. 9, a 100tonne payload transported to Mars utilized laser-driven capsules with 7.5GJ yields and a total number of $8 \times 10^{7}$ targets. Accordingly, scaling our nominal $0.5 \mathrm{GJ}$ hotspot or fast ignition targets by an order of magnitude in yield to 5.6GJ, might require an increase in injected antiprotons for ablative drive by a factor of $\sim 10^{(1+\gamma)^{-1}}$. This translates to $\sim 1.2 \times 10^{16}$ and $\sim 7.6 \times 10^{16} \bar{p}$ for 5.6GJ fast ignition and hotspot capsules, respectively. For mission parameters similar to VISTA, several moles of onboard stored antiprotons might therefore be required

\section{TIMING AND AIMING REQUIREMENTS FOR ANTIPROTON INJECTION}

The maximum antiproton injection time to create the fast ignition hotspot in Section 6 must be of the order of the hotspot disassembly time otherwise fuel expansion will quench the burn before it propagates into the cold fuel. If we represent this by the 
sound transit time across the hotspot at the ignition temperature $T_{i g n}$, then the required injection time is $\tau_{i g n} \sim R_{H S} / c_{S}\left(T_{i g n}\right)$, resulting in a required antiproton injection time of $\sim 25 \mathrm{ps}$. With a required injection of $0.62 \times 10^{15}$ antiprotons into the hotspot (Section 6.4), this represents a peak current of $\sim 3.9 \mathrm{MA}$. Similarly, with a deposition region set to $\sim 2 R_{H S}=2(\rho R)_{i g n}, / \rho_{H S}$, where $\rho_{H S} \approx \rho_{\text {stag }}$, this case requires focal spot diameters of $\sim 30 \mu \mathrm{m}$ on the outside of the compressed fuel. These are formidable requirements for a focused, accelerated beam of $z=1$ particles at kinetic energies of $\sim 1 \mathrm{MeV}$

By contrast, the injection requirements to drive the slow compression phase of fast ignition are considerably relaxed relative to the ignition phase, and also when compared with drive specifications for hotspot ignition under isobaric conditions. In general, comparing the ablative drive requirements for this slow compression phase (Section 6.3) with those for hotspot ignition (Section 5) we would expect the penetration time for the first low intensity shock to be greater because fast ignition capsules would typically be designed with a thicker, more massive shell. Second, the implosion velocities are relaxed, viz. $\approx 1 \times 10^{7} \mathrm{~cm} / \mathrm{s}$ rather than $\approx 3 \times 10^{7} \mathrm{~cm} / \mathrm{s}$ for hotspot ignition. Third, compressed core radii are bigger due to the lower final density requirements, thus shell convergence ratios $C$ will, in general, be lower. Here, we might expect pulse lengths for the slow compression phase for fast ignition to scale as $t \sim C / v$. Accordingly, whereas our $5 \mathrm{mg}$ hotspot targets required a drive pulse length in the vicinity of $\sim 40 \mathrm{~ns}$ (or $\sim 10 \mathrm{~ns}$ at peak power), this relaxes for the slow compression phase for the fast ignition target to $\sim 100 \mathrm{~ns}$ (or $\sim 30 \mathrm{~ns}$ at peak power). With a required injection of $2.5 \times 10^{15} \overline{\mathrm{p}}$ to drive the slow compression phase (Section 6.4), this translates to an antiproton injection current of $\sim 4 \mathrm{kA}$. For the ablative drive phases of both fast ignition and hotspot ignition, the focal requirements are of the order of the target diameter under compression, an increase of some sixty-fold over the antiproton injection focal spot diameter required at the fast ignition hotspot.

In the case of fission-fusion volumetric ignition (Section 4), the antiproton ignition source is applied after the fuel is compressed but, unlike fast ignition, is distributed across the whole compressed core rather than just at the hotspot. Moreover, ignition temperatures can be lower than hotspot-ignited targets while the seeded fuel mass has higher densities; hydrodynamic expansion is correspondingly slower. As above, we assume that this ignition energy is deposited in a time less than the disassembly time of the compressed fuel, requiring an injection pulse length of $\tau \sim R_{f u e l} / c_{s}\left(T_{i g n} f_{u}\right)$ across the compressed fuel radius $R_{\text {fuel }}$. For our design point in Section 4 above for a $5 \mathrm{mg}$ DT fuel mass with, say, a $f_{u}=5 \%$ uranium seed fraction, this translates to $\tau \sim 450 \mathrm{ps}$, intermediate between the injection times for ablative compression and fast ignition of a hotspot. The required antiproton focal spot diameter is of the order of the compressed fuel diameter. i.e., $\approx 2 R_{\text {fuel }}=2(\rho R)_{\text {fuel }} / \rho_{\text {fuel }} \sim 400 \mu \mathrm{m}$.

In summary, total pulse length requirements and, therefore, antiproton injection times for the concepts in this paper scale as:

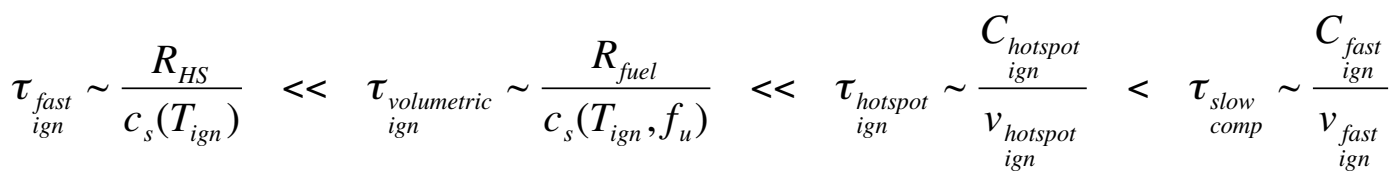


For the 5mg DT target designs above, $\tau_{\substack{\text { fast } \\ \text { ign }}} \sim 25 \mathrm{ps}, \tau_{\substack{\text { volumetric } \\ \text { ign }}} \sim 450 \mathrm{ps}$ (at $f_{u}=5 \%$ ),

$\tau_{\text {hotspot }} \sim 40 \mathrm{~ns}$ (or $\sim 10 \mathrm{~ns}$ at peak power), $\tau_{\text {slow }} \sim 100 \mathrm{~ns}$ (or $\sim 30 \mathrm{~ns}$ at peak power)

Similarly, focal spot diameters for antiproton injection scale as

$d_{\text {fast }} \sim \frac{2(\rho R)_{\text {ign }}}{\rho_{\text {HS }}\left(\approx \rho_{\text {stag }}\right)} \quad<\quad d_{\begin{array}{c}\text { volumetric } \\ \text { ign }\end{array}} \sim \frac{2(\rho R)_{\text {fuel }}}{\rho_{\text {fuel }}} \quad<\quad d_{\begin{array}{c}\text { hotspot } \\ \text { ign }\end{array}} \sim 2 R_{0} / 2 \approx d_{\text {slow }} \sim 2 R_{0} / 2$

For the target design points above, $d_{\substack{\text { fast } \\ \text { ign }}} \sim 30 \mu \mathrm{m}, d_{\substack{\text { volumetric } \\ \text { ign }}} \sim 400 \mu \mathrm{m}, d_{\substack{\text { hotspot } \\ \text { ign }}} \approx d_{\text {slow }} \sim 2000 \mu \mathrm{m}$

\section{ANTIPROTON PRODUCTION, STORGE AND INJECTION}

It is beyond the scope of this paper to formally assess the systems issues pertaining to the production and manipulation of antiprotons for projected applications such as space propulsion. However, given the arguable practicalities for a potential future technology that today is still in a basic science phase [14-29], it behooves us to consider some fundamental requirements. We note that one of us (CDO) has addressed the detailed systems issues for conventional, laser-driven ICF space propulsion [9].

Today, antiprotons are routinely produced at the world's large proton synchrotrons and are subsequently utilized for particle physics research in high energy protonantiproton colliders. Production takes place by accelerating protons onto a cooled tungsten target according to the reaction:

$$
p+p \rightarrow p+p+p+\bar{p}
$$

This minimum set of product particles is necessary for baryon conservation and the threshold energy is $5.63 \mathrm{GeV}$ for a stationary target. For production in a future center-ofmass collider, the threshold would be just twice the proton rest mass, i.e., $1.88 \mathrm{GeV}$. Energies greater than threshold are required for an adequate yield and peak production rates occur around $\sim 200 \mathrm{GeV}$. The resulting antiprotons are directed by a magnetic lens and, following cooling to reduce energy spread, are decelerated to several tens to hundreds of $\mathrm{MeV}$ and collected in storage rings.

The ACOL antiproton production facility at CERN can produce $>10^{12} \overline{\mathrm{p}} / \mathrm{day}$, and the Low Energy Antiproton Ring (LEAR) stores antiprotons at $\sim 200 \mathrm{MeV}$ with a final energy spread of $<<3 \%$ [74]. We note that the goals of the world's high energy physics programs are typically satisfied with a modest production of only $\sim$ few $\times 10^{15} \bar{p} /$ year, about the number required to drive just one high yield ICF target above! Antiproton production efficiency - that is, the ratio of stored antiprotons to accelerated incident protons - on these unoptimized systems is rather low, i.e. $\sim 10^{-4}$. And, given that such antiprotons are employed as mere engineering tools to facilitate particle physics research programs, high production rates are neither desired nor sought. Clearly, for practical applications, a scale-up of production by orders-of-magnitude would be required, 
together with commensurate increases in production efficiencies; candidate methods to achieve this are discussed in Ref. 41. Conceivably, antiproton production for deep space applications could take place in large solar-powered production factories in planetary or solar orbit. However, unlike applications to ICF terrestrial energy production, overall energy breakeven would not necessarily be required.

The kinetic energies required of the antiproton injectors for targets in this paper need only be sufficient for penetration to the required annihilation point, i.e., $\sim 1-10 \mathrm{MeV}$, and are typically less than one percent of the useful fraction of annihilation energy deposited in the targets. Of course, antiproton ion sources would need to be developed. The resulting peak beam currents required are

$$
I_{\bar{p}, \text { beam }}=N_{\bar{p}} e / \tau
$$

where $\tau$ is the required pulse length. This ranges from kiloamperes for direct ablative drive to megamperes for sparking fast ignition hotspots, with intermediate requirements for volumetric ignition targets (Section 7). Although these beams have much lower kinetic energies than typical heavy-ion drivers for ICF [72] and lower currents relative to typical pulsed light ion diodes, the low kinetic beam energy $E_{k}$ will result in a high perveance, $K \sim I_{\bar{p}, \text { beam }} / E_{k}$, that will make it difficult to focus the beam to small spot sizes without neutralized focusing, i.e., without adding a co-moving positively charged beam. Note that neutralizing an antiproton beam would necessitate adding positrons, not electrons; this has been attempted in experiments at CERN [16]. An alternative might be to employ plasma channel focusing through a low density, conventional (normal matter) plasma background. The total injected kinetic energy per target is only a few kilojoules; that is, an average power of a few tens of kilowatts at a target repetition rate of, say, $10 \mathrm{~Hz}$. For space propulsion applications, this minimal recirculating power plus plant auxiliary power requirements could be supplied via a magnetic pickup coil from the main plasma exhaust stream [see, for example, Ref. 9]. Alternatively, for simplicity and minimum mass, a small dedicated fission reactor could be employed.

Antiprotons have been routinely contained in large storage rings for fractions of a year. Low energy antiprotons have also been manipulated and transported in portable Penning Traps with storage times of months [14, 15, 17-19]. Holzscheiter et al. [15] have captured over one million antiprotons from the LEAR ring at CERN in a large Penning trap. Surprisingly, when these antiprotons were cooled to energies significantly below $1 \mathrm{eV}$, the usual determiner of antiproton storage lifetime - i.e., annihilation on residual gas molecules - fell to anomalously low values which may have significance for future long-term storage concepts.

For space applications, antiprotons would be produced in separate, optimized production facilities and the total number required for all targets for a mission would be stored onboard. The limit on the density of antiprotons $n_{\bar{p}}$ stored as a non-neutral, single ion plasma in a confining magnetic field $B$ is set by the Brillouin limit [75]. This expresses the space charge equilibrium condition between the self field of the antiprotons relative to the external field and can be expressed as 


$$
\frac{n_{\bar{p}} m_{p} c^{2}}{B^{2} / 2 \mu_{o}} \leq 1
$$

That is, the applied axial magnetic field containing the radial electric space charge of the non neutral plasma must have an energy density greater than the rest mass energy density of the ions so contained. Brillouin limits arbitrarily close to unity can be obtained in laser-cooled traps at low temperature [75]. As an example, with, say, a nominal $5 \times 10^{15}$ antiprotons required per target and a maximum practical confinement field of, say, 14T, each target requires an antiproton storage volume of $0.009 \mathrm{~m}^{3}$ at the Brillouin limit. Thus, for, say, an inventory of $10^{6}$ targets, the onboard storage volume for antiprotons in single ion form would be $\sim 9 \times 10^{3} \mathrm{~m}^{3}$, that is a vessel some twenty meters on a side. For $\mathrm{Nb}_{3} \mathrm{Sn}$ superconducting magnets and high strength, low temperature stainless steel structure with yield stress of $\sim 800 \mathrm{MPa}$, the vessel mass would be tens of thousands of tonnes assuming a solenoidal trap limited by magnetic hoop stress. This is a significant mass penalty.

The required magnetic confinement field and/or volume and, in particular, vessel mass could be drastically reduced if the antiprotons were stored as a low temperature, neutral plasma of antiprotons and positrons. In this case, the storage limit and required magnetic field would be determined by the macroscopic beta (pressure) equilibrium limit, i.e.

$$
\beta=\frac{2 n_{\bar{p}} k T}{B^{2} / 2 \mu_{o}} \leq 1
$$

where $T=T_{i} \approx T_{e}$ is the plasma temperature. Employment of a field-reversed configuration, might permit beta limits close to unity [56,57]. Note, from Eqs. 26 and 27, that the ratio of the magnetic fields required to contain the antiprotons as a neutral plasma to that required to contain the equivalent number density as a non-neutral plasma is

$$
\frac{B_{\text {neutral }}}{B_{\text {nonneutral }}} \approx \sqrt{\frac{2 k T}{m_{p} c^{2}}}
$$

which, for an ion temperature of, say, $T=10 \mathrm{eV}$, is $\sim 10^{-4}$, thus underlining the value of neutralization. Equivalently, at the same 14T field, the storage volume for the same total $\left(5 \times 10^{15}\right) \times 10^{6}$ antiprotons falls to significantly less than a cubic meter and vessel masses to less than a tonne. An external RF heating source would be required to maintain the temperature of such a neutral plasma against steady-state energy confinement losses.

Antiproton storage in neutral matter form would avoid the high magnetic fields associated with either single ion or plasma containment. Such neutral antimatter would, of course, have to be isolated from normal matter. Experiments at CERN [16] created antihydrogen atoms by traversing a xenon jet with an antiproton beam. Here, a small fraction of the antiprotons convert their energy to electron-positron pairs and a fraction of subsequent antiprotons pick up the positrons. Recently, Gabrielse et al. [17] formed antihydrogen in an excited state by combining antiprotons and positrons in a Penning trap, while Amoretti et al. [18], and subsequently Gabrielse et al. [19,20] detected cold antihydrogen by the same method. 
Long term storage in bulk neutral matter form would require the formation of anti-molecular-hydrogen, i.e. $\overline{\mathrm{H}}_{2}$, and not merely antihydrogen. One method to manipulate bulk antimatter might be in the form of cluster ions, $\overline{\mathrm{H}}_{\mathrm{n}}^{-}$, i.e. a cluster of $\mathrm{n}$ antihydrogen atoms with a positron removed. According to Stwalley [76], formation of anti- $\mathrm{H}$, anti- $\mathrm{H}^{+}$and anti- $\mathrm{H}_{2}-$ could proceed by a number of methods. Molecular antihydrogen, i.e. anti- $\mathrm{H}_{2}$, would be the most difficult of the simple species to form and especially to manipulate because of small magnetic moments and complex vibrational states. However, both the ionic species anti- $\mathrm{H}_{2}{ }^{-}$and anti- $\mathrm{H}_{2}{ }^{+}$would be stable if formed and, in principle, would be more easily controlled and manipulated via magnetic, electrostatic and RF techniques. Antihydrogen ice formation via inverse sublimation would require methods for latent heat removal and nucleation that do not involve a normal matter wall. Levitation of antihydrogen ice would depends on its form -orthohydrogen (spins parallel) or parahydrogen (spins antiparallel). The latter is diamagnetic and might be stably levitated in a static field as has been demonstrated with graphite of similar diamagnetic susceptibility [77]. Active electrostatic levitation with feedback position control might also be performed and would require charging of the ice particles by, say, irradiation with UV light. An analogous trap constructed at the Jet Propulsion Laboratory, Pasadena CA, levitated a $20 \mathrm{mg}$ ball of $\mathrm{H}_{2} \mathrm{O}$-ice in the earth's gravitational field [78].

\section{SUMMARY AND CONCLUSIONS}

In this paper, we have examined the physics underlying the use of antiprotons to drive various classes of ICF targets to high yield by the methods of volumetric ignition, hotspot ignition and fast ignition. We have determined the useable deposition fraction of annihilation energy together with integrated energy balances in the targets and, thereby, deduced the typical number of injected antiprotons required, together with timing and focal spot requirements. A summary is provided in Table 1.

Volumetric ignition with minimum useful yields (few hundred megajoules, $5 \mathrm{mg}$ initial DT mass) results in low gains and requires large numbers of injected antiprotons $\left(\sim 6.5 \times 10^{17}\right)$ in addition to the large precompression energy required to create the initial dense core. This is because the whole compressed fuel is raised to the ignition temperature rather than just a local hotspot as in the other classes of target. Note also that, in addition to the large number of antiprotons required for ignition, they must be focused to a few hundred microns onto a compressed core in a very short time ( $\sim$ few hundred picoseconds). If such targets are to be employed, it appears advantageous to run them in fission-dominated mode, that is with uranium seed fraction above $\sim 15 \%$ and yields in the gigajoule range (for 5mg initial DT mass) - see the footnote to Table 1; the overall gains are, however, still low. Because of the large conventional driver energies required for precompression, and the fact the antiproton number for ablative compression is always lower than that required to promote volume ignition - at least up to a seed fraction of $\sim 25 \%$ - antiproton ablative drive should also be considered for these targets. For

example, at $f_{u}=25 \%$, a total number of $\sim 1.8 \times 10^{18}$ antiprotons would be required $(\sim 50 \%$ going to each of ablative compression and volumetric ignition); the yield would be $\sim 1.5 \mathrm{GJ}$ (70\% fission) and the overall (compression plus ignition) gain is $\sim 3$. 
The fact that it requires significantly more energy to heat fusion fuel relative to compressing it to high density was appreciated early in the ICF program where it was determined that volumetric ignition would probably not lead to high gain fusion. In general, we conclude that $\overline{\mathrm{p}}$-driven fission-fusion volumetric ignition targets offer little promise compared to the other options - that is, pure fusion targets with antiproton ablative drive and burn propagation from a hotspot. Fundamentally, at such low gains, it would seem more expedient to utilize the antiproton annihilation energy independently in the form of directed thrust from the annihilation pions without recourse to fission or fusion

The highest gains in this study occur for antiproton-driven fast ignition (third row of Table 1) but at the expense of two separate $\bar{p}$ injections system, differing in spatial and temporal requirements by two to three orders of magnitude. Here only $\sim 3 \times 10^{15} \overline{\mathrm{p}}$ are required for a yield of $\sim 560 \mathrm{MJ}$ and a gain of several hundred. Scaling this target in yield by an order of magnitude to $\sim 5 \mathrm{GJ}$ would require $\sim 1.2 \times 10^{16}$ injected antiprotons and several moles of stored onboard antiprotons for a Mars roundtrip with a 100tonne payload. However, because the fast ignition system is faced with stringent requirements in terms of injection times and aiming precision - viz., $\sim 25 \mathrm{ps}$ and $\sim 30 \mu \mathrm{m}$, respectively antiprotons appear to have more utility to just drive the much relaxed, slow compression phase. In such case, a separate conventional fast energy source such as a short pulse laser would be needed to spark the ignition.

Of the three target types of this paper, $\overline{\mathrm{p}}$-driven hotspot ignition might appear to offer the best compromise. Several times the delivered antiprotons are required per target relative to fast ignition for a gain of $\sim 100$, but timing ( 40ns) and focal ( 2000microns) requirements are similar to those of the slow compression phase while the short pulse, precise focus constraints required to produce the ignition spot are avoided. Of course, if operated in a direct drive mode, uniform $\overline{\mathrm{p}}$ injection over $4 \pi$ solid angle will be required to avoid low mode drive asymmetries. Alternatively, recourse could be made to semiindirect or indirect drive design with a surrounding hohlraum.

We note that all pulsed space propulsion methods are subject to three constraints if the rocket exhaust is formed by redirection of a plasma by a magnet: (1) the energy in the magnetic field should be at least five times the total energy in the plasma for one pulse, (2) the gyroradii of the individual particles must be considerably smaller than the dimensions of the apparatus, and (3) the dynamics (and primarily the time scale) of the redirection must be short enough to avoid significant Rayleigh-Taylor (interchange) instabilities. To assess the impact of these constraints for any concept, a detailed systems analysis is required, especially to determine the extra mass (expellant) to be added per target to minimize the trip time. Because we are here considering schemes in which most of the target yield arises from fusion and not the antiproton-proton reaction itself (which has long-range products that can affect the size of the expellant mass), there should be no fundamental difference regarding the above constraints whether the drive is antiprotons or, say, lasers. Nevertheless, we emphasize the importance of a detailed systems analysis for any concept that might be proposed based on these results. 
Finally, although antiprotons are truly unique energy vectors, their application to antiproton-driven ICF is speculative. In particular, both the handling of antiprotons and their required injection precision will present significant technical challenges. The storage and manipulation of low energy antiprotons, particularly in the form of antihydrogen, is at present only in a basic science phase; viz., to date, only around one million antiprotons have been stored in Penning traps while antihydrogen - that is a bound state of an antiproton and a positron - has been achieved only fleetingly in such traps. In particular, the total world annual production of antiprotons is of the same order as that required to drive just one of the high gain targets of this paper. Accordingly, novel methods of efficient antiproton production will be required to commence any serious $\mathrm{R} \& \mathrm{D}$ program for this application. Candidate concepts for such scale-up methods are offered in Ref. 41 

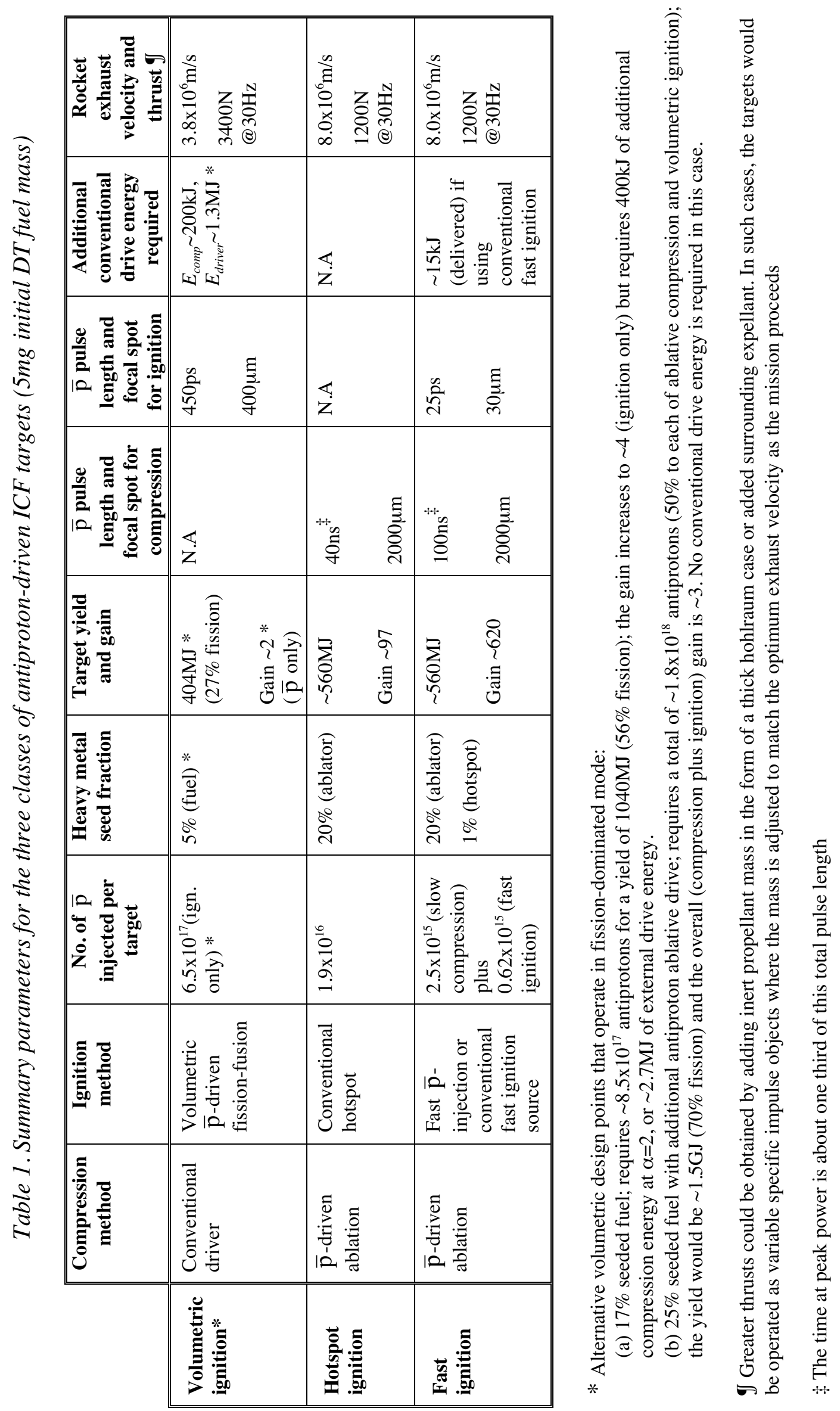


\section{REFERENCES}

1. W.J.Hogan (Ed) "Energy from Inertial Fusion", International Atomic Energy Agency, Vienna, STI/PUB/944, (1995)

2. J.D.Lindl, "Inertial Confinement Fusion: The Quest for Ignition and Energy Gain Using Indirect Drive” (AIP Press, Springer-Verlag, New York, 1998)

3. C.D.Orth, S.A.Payne, W.F.Krupke, Nucl Fusion, 36, 75-116 (1996).

4. R.W.Moir., Fusion Technology 25, 5 (1994) and 30, 1613 (1996)

5. L. J. Perkins, “Nucl. Instr. and Methods in Phys. Research A415 44 (1998)

6. J.D.Sethian et al, "Fusion Energy with Lasers and Direct Drive Targets", accepted for publication in Nucl Fusion (2003)

7. R.A.Hyde, “A Laser Fusion Rocket for Interplanetary Propulsion”, Lawrence Livermore National Laboratory UCRL-88857 (1983)

8. S.K.Borowski, "Comparison of Fusion/Antiproton Propulsion Systems for Interplanetary Travel”, proc $23^{\text {rd }}$ AIAA/SAE/ASME/ASEE Joint Propulsion Conference, San Diego, CA Jun 29-Jul 2, 1987, AIAA-87-1814. Also published as NASA Technical Memorandum 107030 (1987)

9. C. Orth, "VISTA: A Vehicle for Interplanetary Space Transport Application Powered by Inertial Confinement Fusion (Systems Analysis Final Report)," University of California, Lawrence Livermore National Laboratory, UCRL-LR110500 (2003).

10. M. Tabak, J.Hammer, W.Glinsky, W.Kruer, et al., Phys. Plasmas, 11626 (1994)

11. M.C.Herrman, M.Tabak, J.D.Lindl, Phys Plasmas 82296 (2001)

12. W.R.Meier, "Osiris and Sonbrero Inertial Fusion Power Plant Designs" W.J.Schafer \& Asooc., Wakefield MA, WJSA-92-001, US Dept of Energy DOE/ER-54100-1 (1992)

13. E.I.Moses, C.R.Wuest, Fusion Science and Technology, 43420 (2003). See also http://www.llnl.gov/nif/nif.html

14. G.Gabrielse, Phys. Rev. Lett., 272504 (1986).

15. M.H.Holzscheiter., Phys. Lett. A214 279 (1996)

16. G.Baur, Phys Lett, B368, 251 (1996)

17. G.Gabrielese, Phys Lett. B 5071 (2002) 
18. M.Amoretti., Nature, 419456 (2002)

19. G.Gabrielese, Phys. Rev. Lett., 89213401 (2002)

20. G.Gabrielese., Phys. Rev. Lett., 89233401 (2002)

21. G.A.Askaryan, JETP Lett. 17, 424 (1973)

22. F.Winterberg, Z. Naturforsch, 28, 900 (1973)

23. G.A.Smith, p. 296 in Proc. of the RAND Workshop on Antiproton Science and Technology, October 6-9, 1987; B.W. Augerstein et al., (Eds.) (World Scientific, Singapore, 1988).

24. A.Angelopoulos, Phys. Lett. B, 205590 (1988)

25. H.Mancher, Z. Phys. A343 73 (1992)

26. R.A.Lewis, et al., Nucl. Sci.Eng., 109411 (1991)

27. C.E.Gazze et al., Nucl. Sci. Eng., 118217 (1994)

28. I.Kumiko et al., Nucl. Sci. Eng., 118227 (1994)

29 T.Von Edigy et al., Nucl. Phys. A558 383c (1993)

30. W.Sscmid, et al. Nucl. Phys. A569 689 (1994)

31. A.Gsponer, J-P.Hurni, "The Physics of Antimatter-Induced Fusion and Thermonuclear Explosions", p166 in Proc. 4th Intermat. Conf. on Emerging Nucl. Energy Syst., Madrid, Spain, June 1986.

32. A.Gsponer, J-P.Hurni, Atomkernenergie Kerntechnik (Independent Jounal on Energy Systems and Radiation) 49, 198 (1987).

33. R.A.Lewis, "An Antiproton Catalyst for Inertial Confinement Fusion Propulsion", Proc. of AIAA/SAE/ASME/ASEE $26^{\text {th }}$ Joint Propulsion Conf., Orlando, FL July 16181990

34. G.A.Smith, "Antiproton-Catalyzed Microfission/fusion Propulsion Systems for Exploration of the Outer Solar System and Beyond", Technical Interchange Meeting on the Use of Fusion Energy for Space Propulsion", NASA Marshall Space Flight Center, Hunstville AL, March 25-26 1997

35. D.Beller, C.R.Martin, "Proposed Initial Investigation of the Feasibility of Antiproton-Catalyzed Inertial Confinement Fusion". Proc Internat Conf. on Emerging Nuclear Energy Systems, Monterey CA, June 16-21 1991 
36. T.J.Nugent, “A Performance Evaluation of the Ion Compressed AntiprotonCatalyzed Nuclear (ICAN) Propulsion System”, Jet Propulsion Laboratory JPL D8572 (1991)

37 B.R.Wienke “Antiproton Driven Microfission-Fusion on Closer Inspection”, Nuclear Technologies for Space Propulsion NTSE-92, Jackson Hole, WY, American Nuclear Society (1992)

38. G.Gaidos et al. "AIMStar: Antimatter Initiated Microfusion for Precursor Interstellar Missions”, Dept of Physics, Pennsylvania State University, University Park, PA (1998)

39. J.Davis "With Antimatter to the Stars", New Scientist p 66, June 1989

40. R.L. Forward and J. Davis, "Mirror Matter" (Wiley Science Editions, NY) 1988.

41. R.L. Forward, "Antiproton Annihilation Propulsion - Final Report" US Air Force Rocket Propulsion Laboratory AFRPL TR-85-034/055 (1985)

42. D.L. Morgan "Investigation of Matter-Antimatter Interactions for Possible Propulsion Applications" NASA CR-141356 (1974).

43. E. Sanger, Ingenieur-Achiv V. 21213 (1953).

44. D. Dipprey , "Frontiers in Propulsion Research" Jet Propulsion Laboratory JPLTM-33-722 (1975).

45. D.L. Morgan, J. Brit. Interplanetary Soc. 35405 (1982).

46. R.L. Forward, J. British Interplanetary Society 35391 (1982).

47. B. Augerstein, "Some Examples of Propulsion Applications Using Antimatter" RAND Corporation Report P-7113 (1985)

48. S.D. Howe and I.D. Metzger, "Survey of Antiproton-Based Propulsion Concepts and Potential Impact on a Manned Mars Mission" Los Alamos National Laboratory LA-UR-87-2191 (1987).

49. D.L. Morgan, "Antiproton-Matter Interactions in Antiproton Applications", Proc. Workshop on Annihilation in Gases and Galaxies, NASA Goddard Space Flight Center, Greenbelt, MD, July 19-21 1989 , R.J.Drachman (Ed), NASA Conf. Pub 3058 (1990)

50. D.L.Morgan, Hyperfine Interactions 44399 (1988)

51. A.Foderado, "The Elements of Neutron Interaction Theory", p486ff (MIT Press, 1971)

52. M.E.Cuneo, R. Vesey, J.H.Hammer, et al., Laser Particle Beams, 19481 (2001) 
53. S.A.Slutz, M.C.Herrmann, Phys. Plasmas10 234 (2003)

54. R.B. Stephens, Y. Aglitskiy, F. Amiranoff, et al., " $K_{\alpha}$ Fluorescence Measurement of Relativistic Electron Transport in the Context of Fast Ignition" to be published in Phys Rev E (2003)

55. A.Hasegawa., Phys. Rev. Lett. 56, 846 (1986)

56. R.P.Drake, J.H.Hammer, C.W.Hartman, L.J.Perkins, D.D.Ryutov, Fusion Technology 30, 310 (1996)

57 L.J.Perkins, R.P.Drake, J.L.Eddleman, J.H.Hammer, et al., "High Density, High Magnetic Field Concepts for Compact Fusion Reactors", 16th IAEA Fusion Energy Conference, Montreal, Canada, October 1996, IAEA-F1-CN-64/GP-18, (1997)

58. S..E.Jones, Phys. Rev. Lett. 51, 1757 (1983)

59. G.B.Zimmerman, W.L.Kruer, Plama Phys. Controlled Fusion 251 (1975)

60. M.Aguilar-Benitrez et al., "Particle Properties Booklet", American Institute of Physics (1992)

61. D.L.Morgan , V.W.Hughes, Phys Rev. D, 21389 (1970)

62. E.R.Davis, H.C.Metcalfe, J.E.Williams, J.F.Castka. "Modern Chemistry" (Holt, Rineheart and Winston, NY, 2002)

63. H.H.Anderson , J.F.Ziegler, "Hydrogen Stopping Powers and Ranges in All Elements" Vol 3 of The Stopping and Ranges of Ions in Matter (Pergamon Press 1977)

64. M.D.Rosen, J.D.Lindl, A.R.Thiessen “ Model for Gain Versus Laser Energy in Direct Drive Targets”, Laser Program Annual Report - 83, Lawrence Livermore National Laboratory UCRL-500021 (1983)

65. K.Levedahl, J.D.Lindl, Nucl Fusion 37165 (1997)

66. M.M.Basko, J.Johner, Nucl Fusion 381779 (1998)

67. J.Meyer-ter-Vehn, Nucl Fusion 22561 (1982)

68. S.Kahn, R.Harman, V.Forgu, Nucl Sci. Eng. 239 (1965)

69. P.W.Frank, Recoil Range of Fission Fragments" Bettis Atomic Laboratory, Pittsburgh PA, WAPD-BT-30. (1964) 
70. M. Herrmann, Lawrence Livermore National Laboratory. Private communication. (2002) Parameters taken from a 1D LASNEX run for a representative heavy-ion target indirectly-driven by a uniform temperature radiation source with a fusion yield of $\sim 450 \mathrm{MJ}$. The ignition calculations for this class of target are discussed in Ref. 11.

71 S.E. Bodner, D.Gcolombant, J.H.Gardner, R.H.Lemberg, et al., Phys. Plasmas 5 1901 (1998)

72. D.A.Callahan, M.C.Herrmann, M. Tabak, " Laser and Particle Beams, 20405 (2002).

73. R.B.Stephens, S.P.Hatchett, R.E.Turner, "Implosion of an Indirectly-Driven Reentrant Cone Shell Target", Phys Rev Lett., to be published (2003)

74. See, for example, http://www.cern.ch

75. X.-P.Huang, J.J.Bollinger, T.B.Mitchell, W.M.Itano, Phys Rev Lett, 8073 (1998)

76. W.C.Stwalley, p. 373 and 393 in Proc. of the RAND Workshop on Antiproton Science and Technology, October 6-9, 1987; B.W. Augerstein et al., (Eds.) (World Scientific, Singapore, 1988).

77. R.Waldron, Rev. Sci. Instr., 3729 (1966).

78. W.Rhim, "Development of an Electrostatic Levitator at JPL," p.115 in Material Processing in the Reduced Gravity Environment of Space, G. Rindone (Ed.), (Elsevier 1982). 
University of California

Lawrence Livermore National Laboratory

Technical Information Department

Livermore, CA 94551

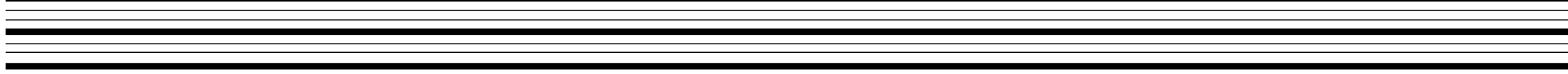

